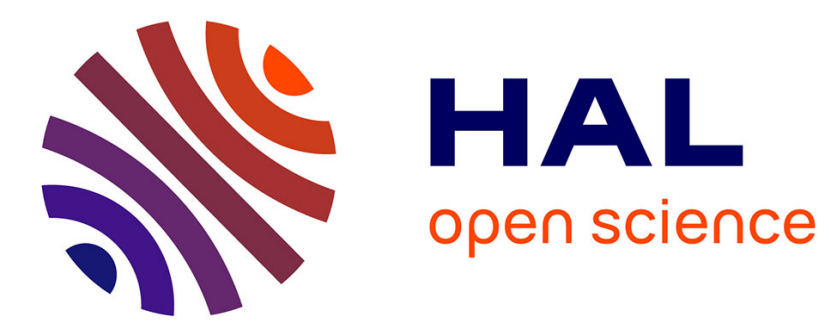

\title{
Contribution of shear strains to the vibrational entropy of defect configurations
}

\author{
Jean-Louis Bocquet
}

\section{To cite this version:}

Jean-Louis Bocquet. Contribution of shear strains to the vibrational entropy of defect configurations. Philosophical Magazine, 2007, 87 (22), pp.3259-3295. 10.1080/14786430701268930 . hal-00513825

\section{HAL Id: hal-00513825 \\ https://hal.science/hal-00513825}

Submitted on 1 Sep 2010

HAL is a multi-disciplinary open access archive for the deposit and dissemination of scientific research documents, whether they are published or not. The documents may come from teaching and research institutions in France or abroad, or from public or private research centers.
L'archive ouverte pluridisciplinaire HAL, est destinée au dépôt et à la diffusion de documents scientifiques de niveau recherche, publiés ou non, émanant des établissements d'enseignement et de recherche français ou étrangers, des laboratoires publics ou privés. 


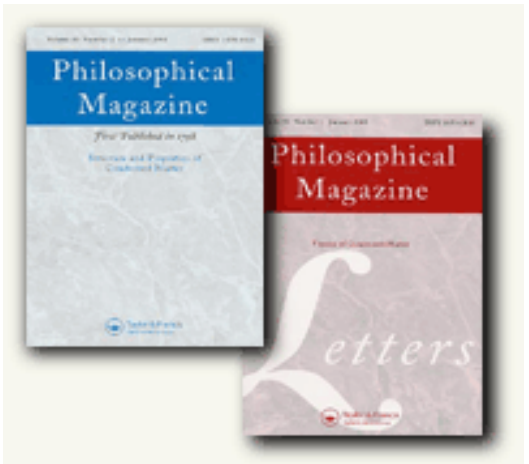

\section{Contribution of shear strains to the vibrational entropy of defect configurations}

\begin{tabular}{|r|l|}
\hline Journal: & Philosophical Magazine \& Philosophical Magazine Letters \\
\hline Manuscript ID: & TPHM-06-Oct-0417.R1 \\
\hline Journal Selection: & Philosophical Magazine \\
\hline $\begin{array}{r}\text { Date Submitted by the } \\
\text { Author: }\end{array}$ & 26-Jan-2007 \\
\hline Complete List of Authors: & BOCQUET, Jean-Louis; CEA-Saclay, DMN / SRMP \\
\hline Keywords: & point defects, vibrational properties \\
\hline Keywords (user supplied): & dilatational strain, shear strain \\
\hline
\end{tabular}

\section{S) ScholaroNE \\ Manuscript Central}


Calculating the vibrational entropy of an $\mathrm{N}$-atom assembly in the harmonic approximation requires the diagonalisation of a large matrix. This operation becomes rapidly time consuming when increasing the dimensions of the simulation cell. In the studies of point defects, a widely used shortcut consists in calculating the eigen modes of the atoms contained in an inner region, called the defect region, while the atoms belonging to the outer region are held fixed, and in applying an elastic correction to account for the entropy stored in the distortion of the outer region. A recent paper proposed to base the correction on the local pressure change experienced by each lattice site. The present contribution is an extension in the sense that it includes the shears. We compared the two approximations for configurations which are currently encountered in defect studies, namely those pertaining to defect formation and migration. The studied defects are the single, diand tri-vacancy as well as the dumbbell interstitial in a host matrix modelled by several empirical potentials mimicking pure copper. It is shown that the inclusion of shears brings a noticeable contribution to the elastic correction for all configurations of low symmetry.

Keywords: vibrational entropy; point defects; shear strains

\section{Introduction}

The calculation of free energy is of great concern in all the fields of materials science. If the evaluation of potential energy has become today a routine task, the evaluation of the entropy remains a difficult one. Ignoring hereafter the configurational and electronic parts of the entropy, the present work will focus on the vibrational contribution in pure substances with or without point defects.

Modern computing facilities have promoted Monte-Carlo techniques: they are today considered as the best candidates for quantitative determinations of freeenergy or free-energy differences, while taking fully into account the detail of the atomic interactions as well as the boundary conditions [1]. Being stochastic in nature, they obtain a result only at the end of a long series of acceptations and rejections; the convergence towards the aimed at quantity with an acceptable uncertainty is never a trivial question and implies a very heavy computational burden. As a consequence their use is most often restricted to those cases where the determination of some basic quantity without any approximation is highly desirable. These highly sophisticated techniques cannot be used routinely whenever a large number of independent calculations is involved in the physical phenomenon to be modelled, like for example diffusive phase transformations described by successive atomic jumps with on-the-fly determinations of the diffusional parameters $[2,3,4]$. This is the reason why alternate and more tractable routes involving less calculations have constantly been looked for, even if the price to be paid for this is, undisputably, some loss of accuracy. In this spirit, the efficiency of harmonic or quasi-harmonic approximations rests on their ability to reduce the initial problem to a deterministic calculation manipulating algebraic objects. They have been extensively explored during decades for studying the formation and the 
migration of point defects, in metallic alloys [5] as well as ionic crystals [6] and have proved their relevance by obtaining in many cases the right order of magnitude for these basic quantities which are accessible to an experimental determination.

At the light of the most recent results, one begins to understand the conditions under which the harmonic (or quasiharmonic) approximation is expected to work. The latter focusses on the curvatures of an harmonic basin of potential energy around some equilibrium state. The situations where the approach departs substantially from more exact evaluations are encountered whenever the temperature raise leads to the emergence of several metastable minima, the energy of which are comparable to the one of the primitive harmonic basin: this case is typically encountered in amorphous systems and metallic nano-aggregates. For specific model systems where all these minima have been detected exhaustively with their accurate Boltzmann weight [7], the harmonic superposition approximation taking into account all these minima with their proper weights does yield an accurate description of the free eneergy over a very large temperature range and predicts transition temperatures with an excellent precision [8]. Coming back to point defect studies, the basic reason why the harmonic or quasiharmonic approximations are so successful is linked to a particularly favourable circumstance: the introduction of a point defect in a pure host matrix is not a perturbation of sufficient extent to question the unicity of the harmonic basin describing the reference state without defect and to lift its degeneracy. As a consequence, following the shift of the eigen modes of this unique basin is sufficient to have a good representation of the free energy changes versus defect introduction or temperature raise. It is worth noticing here that it would not be the case for a concentrated alloy, either crystalline or amorphous. In all what follows we will confine ourselves to the most simple harmonic approximation.

In this frame, the vibrational entropy of a solid containing $\mathrm{N}$ atoms is evaluated as the entropy of $\mathrm{N}$ coupled three-dimensional harmonic oscillators and reads [9] :

$$
\mathrm{S}=\mathrm{k} \sum_{\mathrm{i}=1}^{3 \mathrm{~N}}\left[\mathrm{x}_{\mathrm{i}}\left(\exp \left\{\mathrm{x}_{\mathrm{i}}\right\}-1\right)^{-1}-\log \left(1-\exp \left\{-\mathrm{x}_{\mathrm{i}}\right\}\right)\right]
$$

where $x_{i}=\hbar \omega_{i} / k T$ and the $\omega_{i}$ are the eigen angular frequencies of the system. In the high temperature approximation, the expression reduces to :

$$
\mathrm{S} \square \mathrm{k} \sum_{\mathrm{i}=1}^{3 \mathrm{~N}}\left[1-\log \left\{\mathrm{x}_{\mathrm{i}}\right\}\right] .
$$

The normal modes of this assembly are calculated with the use of the forceconstant matrix $\mathbf{D}$, containing the second derivatives of the potential energy $\mathbf{U}$ versus the displacements of atoms ' $i$ ' and ' $j$ ' of masses $m_{i}$ and $m_{j}$ along the directions ' $\alpha$ ' and ' $\beta$ ' respectively and expressed by:

$$
D_{i j}^{\alpha \beta}=\left[m_{i} m_{j}\right]^{-1 / 2} \frac{\partial^{2} U}{\partial x_{i}^{\alpha} \partial x_{j}^{\beta}} .
$$

\subsection{Supercell method}

The supercell method consists in applying the above equations to a periodically repeated crystallite of a lattice with or without defect. The frequencies $\omega_{i}$ are the phonon modes at zero wave vector in the reduced Brillouin zone corresponding to the large unit cell: three modes have a zero frequency and correspond to the bodily translation induced by the repetition of the simulation cell. After having dropped these three translational modes by deleting the last three rows and columns of $\mathbf{D}$, its 
determinant $\Delta$ is equal to the squared product of the angular frequencies $\omega_{\mathrm{i}}$. In the high temperature approximation, the entropy change is then reduced to:

$$
\mathrm{S}=-\frac{\mathrm{k}}{2} \log (\Delta)+3 \mathrm{k}(\mathrm{N}-1)\left[1-\log \left(\frac{\hbar}{\mathrm{kT}}\right)\right] .
$$

Any modification of the force constants, stemming from a spatial distortion and/or a mass disorder induced by the presence of a point-or-extended defect, produces a corresponding entropy change :

$$
\mathrm{S}^{\prime}-\mathrm{S}=-\frac{\mathrm{k}}{2} \log \left(\Delta^{\prime} / \Delta\right)+3 \mathrm{k}\left(\mathrm{N}^{\prime}-\mathrm{N}\right)\left[1-\log \left(\frac{\hbar}{\mathrm{kT}}\right)\right]
$$

where the primes denote the system with a defect. The last term serves to correct the dimensions of the first term whenever the number N' of atoms in the state with a defect is not equal to that in the reference state, a situation which prevails in all the point defect calculations operating with a constant number of sites.

At last, if the reference state is the perfect crystal, the dimensional correction is commonly carried back onto the perfect contribution by rescaling the number of atoms in the reference state to N':

$$
\mathrm{S}^{\prime}-\mathrm{S} \square-\frac{\mathrm{k}}{2} \log \left(\Delta^{\prime} / \Delta^{\mathrm{N}^{\prime} / \mathrm{N}}\right) .
$$

The calculation of the normal modes implies the diagonalization of the force constant matrix D : even for systems with short-ranged forces, this step remains associated with a large computational cost and to numerical problems, as soon as the simulation cell contains more than a few thousands of atoms. The supercell method is however often kept as a standard reference point for other approximations because its results are known to vary only weakly with the cell size [10]. During the last decades, numerous attempts have continuously searched for various kinds of simplifying approximations.

\subsection{Approximate methods}

These approximations try to reduce the size of the above matrix on the basis of physical arguments: in metals, the interactions are short-ranged thanks to the electronic screening and a small number of neighbour shells is expected to provide the leading contribution to the energy and the entropy.

The Einstein approximation calculates the eigen modes of an assembly of uncoupled particles, where each atom vibrates in the potential well created by its immobile neighbours; the problem is thus reduced to the diagonalisation of $(\mathrm{N}-1)$ $(3 \times 3)$ matrices. Increasing gradually the coupling between the central atom and its neighbours by taking into account more and more distant shells provides a complete hierarchy of local harmonic approximations which produce sensible results for perfect crystals. When applied to the calculation of defect formation entropies $[11,12,13]$ or pre-exponential factors for defect migration [2], these approximations are however unable to capture the quasilocalized modes around the point defect and to offer a quantitative evaluation of entropies.

The second moment approximation postulates a reasonable analytical form for the vibrational local density of states and opens the route towards the determination of temperature dependent equilibrium structures through the direct minimization of a free energy. This formulation was used for the calculation of grain boundary structures as a function of temperature and enlightened the conditions to be fulfilled by any interatomic potential (continuity of third order derivatives): however it does not take into account the off-diagonal elements of the $(3 \times 3)$ force constant matrix and obtains a free energy which is less accurate than the one provided by the local harmonic formulations quoted above $[14,15]$. 
The approximation of the embedded crystallite (also called embedded cluster) consists in partitioning the imperfect crystal into two regions: an inner region containing $\mathrm{N}^{*}$ defect site(s) with a selected set of neighbours and an outer region containing the $\mathrm{N}-\mathrm{N}^{*}$ remaining atoms. Two routes have been followed then:

i) the Green function method is semi-analytical and assumes that the force constants are changed only in the inner region. Several treatments can be done according to whether the atomic positions used for the calculation of force constants are the perfect positions, or the fully relaxed ones, thus producing results of increasing accuracy [16]. In ionic crystals, the problem is further complicated by several features which are specific to ionic bonding, namely the long-ranged forces and the alternate charges of successive shells [17]. The pedagogical interest of Green function formulations is obvious since tractable analytical expressions can be obtained under simplifying assumptions; however, quantitative results are obtained only when the fully relaxed positions of a large number of neighbours are used, which forbids any analytical formulation of the solution and weighs the computational burden down by a appreciable amount;

ii) the brute force method rests entirely on the knowledge of interatomic interactions as a function of distance. As a first step the fully relaxed positions are determined for all atoms in the cell; as a second step, the vibrational modes of the atoms belonging to the inner region are calculated, while keeping immobile the atoms of the outer region. It can be shown that the contribution of the outer region to the entropy must scale as $1 / \mathrm{N}^{*}$ where $\mathrm{N}^{*}$ is the number of atoms in the inner region [16]. Two approaches have been tried: a first procedure determines the limit of the entropy when $\mathrm{N}^{*}$ is increased, but the latter must be limited by a moderate upper bound in order to keep the computational work to a tractable level [18]; a second procedure (named 'elastically corrected embedded cluster') keeps an inner zone of moderate extension but applies systematically an elastic correction to account for the entropy change stemming from the lattice distortion of the outer region [19].

In its original formulation, the approximation of the elastically corrected embedded cluster is convenient for defects of spherical symmetry like a vacancy: indeed, through the change of local pressure on each site, a corresponding local expansion or contraction is deduced and further conveniently translated into an entropic contribution. This procedure improves noticeably the value of the total entropy [19]. However the shear components of the local distorsion become no longer negligible for more complex defect configurations like defect clusters or saddle point configurations, the dipolar tensors of which exhibit three very different eigen values, whatever the type of potential (pairwise, many-body) used in the calculations [20-23]. As an illustration, the saddle configuration for a vacancy jump in a face-centered cubic lattice along $<110>$ exhibits an orthorhombic symmetry, corresponding to a contraction of the lattice along $<110>$ together with an extension along $<001>$; depending on the detail of the potential, another contraction of smaller magnitude along $<1-10>$ can be observed [21].

The aim of the present paper is to go one step further along the line initiated in [19] and to propose a more refined method including the contribution of shear strains to vibrational entropies in the frame of the harmonic approximation. The paper is organized as follows: i) section 2 recalls some basics about the link between strain, energy and entropy and defines the ingredients to be determined, namely the entropic constants; ii) section 3 explains the way how the entropic constants are practically evaluated and how they will be used in an heterogeneously strained medium; iii) section 4 illustrates this approach on copper, when modelled with several empirical potentials available in the literature. Various analytic forms 
will be chosen with the hope of extracting generic features; we show in passing that the continuity of all the ingredients entering the formulation of the interactions and of their derivatives up to third order included is mandatory. In order to make the paper not too obscure, only the main lines of the approach are described and presented in the body of the text, while relegating all technical details in appendices.

\section{Energy and entropy change under a uniform strain}

\subsection{Energy change and elastic constants}

For a uniformly strained medium, the energy increase per unit volume, under zero external pressure and at $0 \mathrm{~K}$, is given by:

$$
\Delta \mathrm{U}=\frac{1}{2} \mathrm{C}_{\mathrm{ijkl}} \eta_{\mathrm{ij}} \eta_{\mathrm{kl}}+\frac{1}{6} \mathrm{C}_{\mathrm{ijklmn}} \eta_{\mathrm{ij}} \eta_{\mathrm{kl}} \eta_{\mathrm{mn}}+\frac{1}{24} \mathrm{C}_{\mathrm{ijklmnop}} \eta_{\mathrm{ij}} \eta_{\mathrm{kl}} \eta_{\mathrm{mn}} \eta_{\mathrm{op}} \cdots
$$

where $\mathrm{C}_{\mathrm{ijkl}}, \mathrm{C}_{\mathrm{ijklmn}}, \mathrm{C}_{\mathrm{ijklmnop}}$ are the second, third, fourth ... order Brugger elastic constants [24] and $\eta_{\mathrm{ij}}$ the components of the Lagrange strain tensor. Using hereafter Voigt's contraction of indices (ii $=>\mathrm{i} ; 23=>4 ; 31=>5 ; 12 \Rightarrow 6$ ) and limiting ourselves to cubic symmetry for sake of simplicity, the summation can be recast under a standard form displaying only the independent elastic constants, namely the three ones of second order, the six ones of third order and the eleven ones of fourth order [25], according to:

$$
\begin{aligned}
\Delta \mathrm{U} & =\frac{1}{2} \mathrm{C}_{11} \mathrm{P}_{11}+\mathrm{C}_{12} \mathrm{P}_{12}+\frac{1}{2} \mathrm{C}_{44} \mathrm{P}_{44} \\
& +\frac{1}{6} \mathrm{C}_{111} \mathrm{P}_{111}+\frac{1}{2} \mathrm{C}_{112} \mathrm{P}_{112}+\mathrm{C}_{123} \mathrm{P}_{123}+\frac{1}{2} \mathrm{C}_{144} \mathrm{P}_{144} \\
& +\frac{1}{2} \mathrm{C}_{155} \mathrm{P}_{155}+\mathrm{C}_{456} \mathrm{P}_{456}+\frac{1}{24} \mathrm{C}_{1111} \mathrm{P}_{1111}+\frac{1}{6} \mathrm{C}_{1112} \mathrm{P}_{1112} \\
& +\frac{1}{4} \mathrm{C}_{1122} \mathrm{P}_{1122}+\frac{1}{6} \mathrm{C}_{1123} \mathrm{P}_{1123}+\frac{1}{4} \mathrm{C}_{1144} \mathrm{P}_{1144}+\frac{1}{4} \mathrm{C}_{1155} \mathrm{P}_{1155} \\
& +\frac{1}{2} \mathrm{C}_{1244} \mathrm{P}_{1244}+\frac{1}{2} \mathrm{C}_{1266} \mathrm{P}_{1266}+\mathrm{C}_{1456} \mathrm{P}_{1456}+\frac{1}{24} \mathrm{C}_{4444} \mathrm{P}_{4444} \\
& +\frac{1}{4} \mathrm{C}_{4455} \mathrm{P}_{4455}+\ldots .
\end{aligned}
$$

The $\mathrm{P}_{\mathrm{ij}}, \mathrm{P}_{\mathrm{ijk}}, \mathrm{P}_{\mathrm{ijkl}}$ are homogeneous polynomials of second, third and fourth order respectively of the Lagrange strains $\left\{\eta_{i}\right\}$. Their expressions can be found in [26] up to third order and in [27] for fourth order (apart from an error in some multiplicative factors ahead); they are recalled below in equations 9 for sake of completeness. 
$\mathrm{P}_{1144}=\eta_{1}^{2} \eta_{4}^{2}+\eta_{2}^{2} \eta_{5}^{2}+\eta_{3}^{2} \eta_{6}^{2}$

$P_{1155}=\eta_{1}^{2}\left(\eta_{5}^{2}+\eta_{6}^{2}\right)+\eta_{2}^{2}\left(\eta_{6}^{2}+\eta_{4}^{2}\right)+\eta_{3}^{2}\left(\eta_{4}^{2}+\eta_{5}^{2}\right)$

$P_{1244}=\eta_{1} \eta_{2}\left(\eta_{4}^{2}+\eta_{5}^{2}\right)+\eta_{2} \eta_{3}\left(\eta_{5}^{2}+\eta_{6}^{2}\right)+\eta_{3} \eta_{1}\left(\eta_{6}^{2}+\eta_{4}^{2}\right)$

$P_{1266}=\eta_{1} \eta_{2} \eta_{6}^{2}+\eta_{2} \eta_{3} \eta_{4}^{2}+\eta_{3} \eta_{1} \eta_{5}^{2} \quad P_{1456}=\left(\eta_{1}+\eta_{2}+\eta_{3}\right) \eta_{4} \eta_{5} \eta_{6}$

$\mathrm{P}_{4444}=\eta_{4}^{4}+\eta_{5}^{4}+\eta_{6}^{4} \quad \mathrm{P}_{4455}=\eta_{4}^{2} \eta_{5}^{2}+\eta_{5}^{2} \eta_{6}^{2}+\eta_{6}^{2} \eta_{4}^{2}$

The elastic constants can be calculated to any order through lattice sums as soon as the interatomic potential is known, provided its analytical expression is everywhere continuous up to the necessary order (third order at least for $\mathrm{C}_{\mathrm{ijk}}$, fourth order at least for $\mathrm{C}_{\mathrm{ijkl}}$...etc) [28]. We report in Appendix A the formal expressions of the fourth and fifth order elastic constants as a function of the derivatives of the pair and $\mathrm{N}$-body terms which will be used later on for the potentials under examination.

For sake of simplicity in its further use, the equation 8 is rewritten in a more compact form. Renaming the 20 elastic constants by $C_{i}\left(C_{11}=C_{2}, C_{12}=C_{3}, C_{44}=\right.$ $\mathrm{C}_{4} \ldots$..etc $)$ and the 20 homogeneous polynomials by $\mathrm{P}_{\mathrm{i}}\left(\mathrm{P}_{11}=\mathrm{P}_{2}, \mathrm{P}_{12}=\mathrm{P}_{3}, \mathrm{P}_{44}=\mathrm{P}_{4}\right.$ ...etc) in the order of their introduction in equation 8 (including the fractional multiplicative term), the equation is transformed into:

$$
\Delta \mathrm{U}=\sum_{\mathrm{k}=2}^{21} \mathrm{C}_{\mathrm{k}} \mathrm{P}_{\mathrm{k}}
$$

\subsection{Entropy change and entropic constants}

The internal energy and entropy of a crystalline body must be represented by functional forms which are invariant through the symmetry operations of the lattice [29]; their expressions are functions of the same combinations of scalar invariants, the set of which is known for all lattices and symmetry groups [30]. As a consequence, the general expression for the entropy change under an homogeneous strain will look like the one for the energy, where the elastic constants $C_{i j}, C_{i j k}$, $\mathrm{C}_{\mathrm{ijkl}}$ will be replaced by 'entropic constants' $\lambda_{\mathrm{ij}}, \lambda_{\mathrm{ijk}}, \lambda_{\mathrm{ijkl}}$. A difference must be noticed however. Concerning the energy, the reference state is usually taken as the equilibrium state under zero external pressure, the latter condition bringing no further contribution to the energy if the volume is increased during the application of the strain; therefore, the first order term which is proportional to the volume expansion is lacking in equation 8. Conversely, when considering the entropy, the 
dilatational part of the strain brings always a non-vanishing first order contribution proportional to the volume expansion [9]:

$$
\partial \mathrm{S} /\left.\partial \mathrm{V}\right|_{\mathrm{P}}=\alpha \mathrm{B}
$$

where $\alpha$ is the thermal volume expansion coefficient, B the bulk modulus. Switching from derivatives to finite differences and denoting the volume of the system in the reference state by $\mathrm{V}_{\mathrm{o}}$, an alternate writing of this equation enlightens the link existing between the entropy change and any volume change in general, whatever its physical origin (temperature increase, elastic strain ...) :

$$
\Delta \mathrm{S}=\alpha \mathrm{BV}_{\mathrm{o}} \text { dilat }=\lambda_{1} \text { dilat }
$$

Let us recall here that in Lagrange's formalism, the volume expansion denoted here by 'dilat' is no longer expressed as the trace of the strain tensor but rather by:

$$
\text { dilat }=[\operatorname{Det}(\mathbf{I}+2 \boldsymbol{\eta})]^{1 / 2}-1
$$

As above for the energy, the entropy change is written in a closed form. Renaming by $\lambda_{\mathrm{k}}(\mathrm{k}=1,21)$ the entropic constants and by $\mathrm{P}_{1}$ the volume expansion given by equation 13 , the entropy change will later on be expressed as:

$$
\Delta \mathrm{S}=\sum_{\mathrm{k}=1}^{21} \lambda_{\mathrm{k}} \mathrm{P}_{\mathrm{k}}
$$

\section{Determination of entropic constants}

The free energy of a system submitted to specific boundary conditions (external stress, temperature) can be determined in an elegant way by a direct minimisation of the total free energy yielding the equilibrium atomic configuration [31, 32]. The vibrational contribution to the quasiharmonic free energy is given as a function of an arbitrary wave vector $\mathbf{q}$ but a numerical summation over a relevant set of $\mathbf{q}$ vectors within the first Brillouin zone is then necessary to obtain the final value of the entropy as a function of the strain amplitude. Since we are working in the harmonic approximation with a perfect system containing one single atom per unit cell, a simpler method is sufficient: the homogeneous strain applied to the simulation cell involves no change of the inner atomic coordinates and the relaxation step becomes superfluous. This is the reason why we choose a direct route: a set of predetermined strains of increasing amplitudes are imposed to the simulation cell and the entropy changes are numerically evaluated and collected; the desired entropic constants are then extracted through a least square fitting of the data to the theoretical behaviour depicted by equation 14 .

\subsection{Definition of the set of strains}

Following Cousins [26] we define a stretching tensor $\mathbf{J}$ which is chosen rotation free by selecting a symmetrical form. The intensity of the components can be varied at will with respect to some arbitrary amplitude denoted hereafter by $\varepsilon$ :

$$
\mathbf{J}=\left|\begin{array}{ccc}
1+\mathrm{d}_{1} \varepsilon & \mathrm{d}_{6} \varepsilon & \mathrm{d}_{5} \varepsilon \\
\mathrm{d}_{6} \varepsilon & 1+\mathrm{d}_{2} \varepsilon & \mathrm{d}_{4} \varepsilon \\
\mathrm{d}_{5} \varepsilon & \mathrm{d}_{4} \varepsilon & 1+\mathrm{d}_{3} \varepsilon
\end{array}\right|
$$

The Lagrangian strain tensor $\boldsymbol{\eta}$ is then defined by $\boldsymbol{\eta}=\frac{1}{2}\left(\mathbf{J}^{2}-\mathrm{I}\right)$ where $\mathbf{I}$ stands for the unit $(3 \times 3)$ matrix. A volume expansion is involved whenever the determinant $\operatorname{Det}(\mathbf{J})$ is larger than unity and a contraction otherwise. As a consequence, for a 
given $\mathbf{J}$, the strains defined by $\mathbf{J}\left(\operatorname{Det}(\mathbf{J})^{-1 / 3}\right.$ or $\mathbf{J}$ will take place at constant volume or not, respectively. We gathered and numbered in table 1 the set of strains which were applied to the simulation cell. Some of the strains are pure shears inducing no volume change $\left(\mathrm{n}^{\circ} 2,4,6,9-26,28,30,32,34\right)$; several others imply a volume change of first order $\left(n^{\circ} 1,3,7,29,31,33,35\right)$ or second order $\left(n^{\circ} 5,8,27\right)$ with respect to $\varepsilon$. Several of them are redundant (11 and 12, 15 and 16 ...etc) and are expected to give similar results (they were subsequently checked that they do so). Many of these strains would be impossible to apply in a practical experiment, at variance with the simulation of a true elastic axial loading [33]: they must be viewed as thought experiments which are used as a simple and systematic way to probe numerically the entropy surface as a function of the strain variables $\left\{\eta_{i}\right\}$.

(insert table 1 around here)

The strains are applied to the three orthonormal basis vectors $(\mathbf{a}, \mathbf{b}, \mathbf{c})$ defining the edges of the simulation box. As already mentionned above, the symmetry of the system implies that the reduced atomic coordinates need not to be changed; as a matter of fact, the conjugate gradient algorithm in charge of relaxing the atomic positions finds systematically that the system is already in its minimum energy. The product of the eigen modes is then determined in the deformed state, which involves only the evaluation of the determinant of the force constant matrix. The corresponding energy and entropy changes with respect to the perfect undeformed state are recorded.

The calculations are conducted in a cubic cell of FCC structure containing 864 atoms. To gather a significant amount of results, the amplitude $\varepsilon$ of the strain is varied in the range $\left[-10^{-2}:+10^{-2}\right]$ with 100 equally spaced mesh points between $[ \pm$ $\left.10^{-2}: \pm 10^{-4}\right]$ and 100 between $\left[ \pm 10^{-4}: \pm 10^{-6}\right]$ for each type of strain.

\subsection{Extraction of the entropic constants}

Before extracting the entropic constants by a least square fitting of equation 14 , a preliminary check was performed on the elastic constants in order to assess the relevance of the set of strains used for the measurements.

Equation 7 is a power series expansion around the undeformed reference state: the coefficients are the equilibrium elastic constants of increasing order which are obtained on the perfect lattice through lattice sums (cf. [28] and Appendix A).

The same equation can be used for finite (but still small) strains: to reach a quantitative precision for finite strains, a sufficient number of terms must be taken into account: the larger the strain amplitude, the higher the number of terms. The latter is determined empirically through the comparison of the energy change which is directly measured on the simulation cell and the energy change predicted by equation 7 with the elastic constants at equilibrium : for a strain amplitude of the order of $10^{-6}$, it is checked that the energy change which is numerically detected on the cell is perfectly accounted for by equation 7 (or 10) with the second order terms only; conversely for strain amplitudes amounting up to a fraction of a percent, it can be checked that the fourth order terms in equation 7 (or 10) are necessary. It was further checked that fifth order terms did not bring a further detectable contribution. This is the reason why we used systematically the fourth order terms to account for the energies of the whole set of deformed states. The simulated values were reproduced by equation 10 with an average relative precision better than $10^{-5}$.

As a further check, the elastic constants which were determined at rest through lattice sums were compared with the elastic constants obtained by fitting the whole 
collection of energy changes measured on the deformed cell under strains of finite amplitude (equation 10). The agreement between the two is excellent as displayed in table 2 for the potential FS-D-2.82 described in more detail in appendix B . As expected, the tightness of the agreement depends on the order: the elastic constants fitted on finite strain results agree with their lattice sums counterparts to better than $10^{-4}, 10^{-3}$ and a few $10^{-2}$ for second, third and fourth order constants respectively. The fact that all elastic constants were reasonably reproduced was interpreted as an indication that the set of strains was broad enough to probe correctly the potential energy surface of deformed states. This conclusion was assumed to hold also for the entropies, where such a direct check was not possible.

The corresponding entropic constants were then determined in the same way by fitting the entropic changes to equation 14 with a relative accuracy of the same order as that observed for the energy. The entropic constants exhibited however a higher sensitivity to the fitting procedure than the elastic ones. For instance $\lambda_{1}$ and $\lambda_{4}$ are rather insensitive to the maximum order retained in equation 14 or to the strain amplitudes used in the fitting, whereas $\lambda_{2}$ and $\lambda_{3}$ could vary of $20 \%$. This is the reason why we consider the entropic constants as defined to no better than $10^{-4}$ for the first order constant $\lambda_{1}$, a few $10^{-2}$ for the second order constants $\lambda_{2}=\lambda_{11}$, $\lambda_{3}=\lambda_{12}$ and $\lambda_{4}=\lambda_{44}$ and a few $10^{-1}$ for the third order ones $\lambda_{5}$ to $\lambda_{10}$. The fourth order ones could not be determined with a sufficient precision.

\subsection{Use of the entropic constants in defect configurations}

We report first the way how the coefficients in equation 14, which were determined for homogeneously strained systems, can be used for the heterogeneous deformations taking place around point defects. We then discuss the underlying assumptions and justify the calculation procedure.

3.3.1 Procedure used for the calculation. Firstly, we assume that the same type of formulation can be applied locally, that is, on each of the $\mathrm{N}-\mathrm{N}^{*}$ sites of the outer region where the values of the polynomials $\mathrm{P}_{\mathrm{k}}$ will now depend on the local strain measured on site ' $\mathrm{i}$ '. The total entropy change $\Delta \mathrm{S}_{\text {out }}$ of the outer region will thus include a summation running over the $\mathrm{N}-\mathrm{N}^{*}$ lattice sites:

$$
\Delta \mathrm{S}_{\text {out }}=\sum_{\mathrm{i}=\mathrm{N}^{*}+1}^{\mathrm{N}} \sum_{\mathrm{k}=1}^{21} \lambda_{\mathrm{k}}\left[\mathrm{P}_{\mathrm{k}}\right]_{\mathrm{i}}=\sum_{\mathrm{i}=\mathrm{N}^{*}+1}^{\mathrm{N}}\left[\Delta \mathrm{S}_{\text {out }}\right]_{\mathrm{i}}
$$

where $\left[\Delta S_{\text {out }}\right]_{i}$ is the contribution of atom on site ' $i$ ' to the entropy change.

* secondly, we propose a consistent and stable procedure for determining the local strain. At the end of a relaxation calculation, the energy of the system with a defect is known with a precision of the order of $10^{-12}$ at best and the atomic coordinates are not known to better than $10^{-6}$. The local strains must then be larger than a few $10^{-6}$ to be detected with some confidence.

Using the atomic coordinates to calculate the local strain is not convenient: the discrete and anisotropic nature of the lattice at short scale complicates the calculation of the distortions and numerical derivatives of the various displacements must be taken, leading to an important numerical noise. We propose alternatively to evaluate the atomic stresses according to their thermodynamical definition, i.e. as the derivatives of the energy contribution of atom ' $i$ ' with respect to the local strains [34]; the calculation of the atomic stresses is straightforward 
[35] and gives access to the local strains. The calculated strain in this procedure becomes much less sensitive to the numerical noise on individual atomic coordinates since it rests on some average performed over all the neighbours interacting with a given atom. We recall below the usual way of determining numerically the stress components and establish the formal expression of stress versus strain components.

The interactions model the total energy of the atomic assembly as well as the energy per atom 'i' by:

$$
\mathrm{U}=\frac{1}{2} \sum_{\mathrm{i}} \sum_{\mathrm{i} \neq \mathrm{j}} \mathrm{V}\left(\mathrm{r}_{\mathrm{ij}}\right)-\sum_{\mathrm{i}} \mathrm{F}\left(\rho_{\mathrm{i}}\right)=\sum_{\mathrm{i}} \mathrm{U}_{\mathrm{i}}
$$

where $V\left(\right.$ rij) stands for the pairwise part, $F$ for the embedding function and $\rho_{\mathrm{i}}$ is a local density using a pairwise function $\Phi\left(\mathrm{r}_{\mathrm{ij}}\right)$ and given by :

$$
\rho_{\mathrm{i}}=\sum_{\mathrm{i} \neq \mathrm{j}} \Phi\left(\mathrm{r}_{\mathrm{ij}}\right)
$$

The component of the local stress on site ' $\mathrm{i}$ ' is given by:

$$
\sigma_{\alpha \beta}^{\mathrm{i}}=-\frac{1}{\Omega_{\mathrm{i}}} \frac{\partial \mathrm{U}_{\mathrm{i}}}{\partial \eta_{\alpha \beta}}=-\frac{1}{2 \Omega_{\mathrm{i}}} \sum_{\mathrm{j} \neq \mathrm{i}}\left(\frac{\partial \mathrm{V}}{\partial \mathrm{r}_{\mathrm{ij}}}-\frac{\partial \mathrm{F}}{\partial \rho_{\mathrm{i}}} \frac{\partial \Phi}{\partial \mathrm{r}_{\mathrm{ij}}}\right) \frac{\mathrm{r}_{\mathrm{ij}}^{\alpha} \mathrm{r}_{\mathrm{ij}}^{\beta}}{\mathrm{r}_{\mathrm{ij}}}=\sigma_{\mathrm{I}}^{\mathrm{i}}
$$

where $\Omega_{\mathrm{i}}$ stands for the atomic volume attached to site ' $\mathrm{i}$ ', $\mathrm{r}_{\mathrm{ij}}^{\alpha}$ for the ' $\alpha$ ' component of vector $r_{i j}$ and 'I' stands for Voigt's index ' $\alpha \beta$ '. All these quantities can be numerically determined for each site ' $i$ ' ; further, the stress components were derived with $\Omega_{\mathrm{i}}$ set equal to the average atomic volume $\Omega_{\mathrm{o}}$. We checked later that using the volume of the Voronoi polyhedron of each atom in place of the average atomic volume did not change the resulting values noticeably, due to the smallness of the strains coming into play.

The theoretical expression of the stress component is readily obtained by deriving equation 10 :

$$
\sigma_{\mathrm{I}}^{\mathrm{i}}=\sum_{\mathrm{k}=2}^{21} \mathrm{C}_{\mathrm{k}}\left[\frac{\partial \mathrm{P}_{\mathrm{k}}}{\partial \eta_{\mathrm{I}}}\right]_{\mathrm{i}}
$$

The presence of higher order terms forbids a linear inversion of equations 20 . The components of the local strain are thus adjusted by a conjugate gradient procedure minimising the sum of the squared departures between equations 19 and 20 . The solution of the linearised problem, resting on the use of only $\mathrm{C}_{11}, \mathrm{C}_{12}$ and $\mathrm{C}_{44}$, is taken as the starting point of the iterative process.

It is worth noticing here that the approach described in [19] assumed a linear relationship between stress and strain; this assumption allowed to convert directly the local pressure change $\Delta \mathrm{p}_{\mathrm{i}}$ into a local volume change, which was translated afterwards into a local entropic contribution through equation 12 :

$$
\Delta \mathrm{S}_{\mathrm{out}}=-\frac{\lambda_{1}}{\mathrm{~B}} \sum_{\mathrm{i}=\mathrm{N}^{*+1}}^{\mathrm{N}} \Delta \mathrm{p}_{\mathrm{i}}
$$

where the summation runs on the $\mathrm{N}-\mathrm{N}^{*}$ atoms of the outer region. Since our relaxations are performed under zero external pressure, equation 21 can be written in an alternate form : the average internal pressure calculated from a summation over the internal stresses being zero, the summation above can be replaced by a summation with an opposite sign running only on those atoms of the inner region: 
3.3.2 Discussion about the underlying assumptions. We discuss below the relevance of equations 16 to 20 to our problem, namely the introduction of a local entropy per site, the use of a stress tensor defined at the atomic level and finally the neglectance of any strain gradient contribution. For this purpose, we will make use of the results obtained in a cell containing 500 atoms for convenience, and interacting through the potential FS-Barreteau described in Appendix B.

* Equation 16 assumes that the total entropy increase can be decomposed into a sum of local entropies attached to each atom. This idea is widely accepted today since a local density of vibrational states can be defined by a projection operation : for each vibrational mode $\omega_{\mathrm{j}}$, the contribution of atom ' $\mathrm{i}$ ' is given by to mode ' $\mathrm{j}$ ' is given by $\sum_{\alpha}\left|\mathrm{V}_{\mathrm{j} \text {,i } \alpha}\right|^{2}$ where $\mathbf{V}_{\mathrm{j}}$ is the normalized eigen vector attached to $\omega_{\mathrm{j}}$ and $\mathbf{V}_{\mathrm{j}, \mathrm{i} \alpha}$ its three components pertaining to atom 'i' $(\alpha=1,2,3)$. The local entropy increment $[\Delta \mathrm{S}]_{\mathrm{i}}^{\text {proj }}$ brought by the atom ' $\mathrm{i}$ ' in the configuration containing a vacancy or a migrating atom at its saddle position can be calculated in a cell where all atoms are allowed to vibrate (the subscript 'out' will be dropped for brevity in what follows). These quantities are stored and used later to rate the values proposed by the elastic correction.

** Equation 16 relies implicitely on the fact that a local distorsion can be defined in the configurations containing a defect. Let us emphasize first that the assumption is far from being obvious: it is not granted at all that the result of a static relaxation process, which is free to move the $\mathrm{p}$ neighbours of a given atom in all directions for the purpose of an energy minimization (3p independent coordinates) can be reasonably accounted for by a mere distortion having only 6 independent components. It will be seen later when analyzing the results that it is however reasonably so.

Any local quantity depending on the local strain could be used to extract the strain components : for instance, with the interatomic potentials under examination, the energy could be a valuable candidate since it has been defined locally thanks to equation 17. Indeed, it is commonly used to explore energy changes versus lattice expansion or contraction, but it is not a sensitive detector of a more complex local deformation because it takes too rough an average which levels off the differences between extended and compressed bonds and is insensitive to bond angles. As an illustration, sites with a cohesive energy larger than that of the bulk were found in a grain boundaries, although the local atomic density is lower, as well as in the vicinity of a free surface [36-38]: the corresponding energy change with respect to 
the undeformed bulk being negative, it cannot be accounted for by equation 8 since the latter predicts only positive changes.

The local atomic stress, as defined above, seems to be more convenient: being tensorial with the same number of components as the strain, it keeps a better track of the relative distorsion of neighbouring bonds than the energy. The thermodynamical definition at work in equation 19 can be used on each site as soon as the energy per site can be defined, which is the case for the potentials used in the present study. This local stress reflects the state of the surroundings and keeps a detailed account of the discrete nature of matter at the atomic level (changes in bond lengths and of their relative orientations): as a consequence, its rapid variation from one site to another in the vicinity of a discontinuity is not surprising. It is well known that the relaxation of atomic planes parallel to a free surface can be observed inward or outward, depending on the potential, and that the sign of the relaxation can pretty well not be uniform and change from one plane to the next: all these features are involved in the calculation of the stress components at the atomic level, and the oscillations close to a free surface reflect these changes of the local neighbourhood $[39,40]$. It is understandable that the identification of this local thermodynamical stress with the mechanical stress as defined by Cauchy is possible only after some coarse-grain average over a region of sufficient extent has been performed, in order to wipe out all these local variations which can be neither understood nor accounted for by continuum mechanics. Several types of coarse graining have been proposed in the past [41-43], but it is worth noticing that all of them imply in their definition, the use of a bond function which is in charge of partitioning the energy carried by each bond between the inner and the outer part of the grain: the underlying geometric definition seems at least as arbitrary and questionable as the volume $\Omega_{i}$ used in equation 19. Incidentally, it should be mentionned that the debate about the inclusion (or not) of the momentum term in the expression of the stress [43] is irrelevant in the present case, since only static relaxations are performed.

In the present study, the only ingredient which is actually looked for is a quantitative information which allows us to go back to the local strain as precisely as possible. The fact that equation 19 represents a true mechanical stress or not is of no concern : the only important point is the fact that the information on the local strain is more faithfully transmitted by the thermodynamical stress defined on each atomic site than by a mechanical stress which implies a coarse-graining. To prove the relevance of the local atomic stresses as defined by equation 19 for the present problem, the following calculations were done. First, the quantities $\left[\Delta S_{\text {out }}\right]_{i}^{\text {proj }}$ were calculated through the determination of the eigenmodes $\omega_{j}$ and the corresponding eigenvectors in the perfect and imperfect configurations, when all atoms in the cell were allowed to vibrate. Then the entropy increments predicted by the elastic correction $\left[\Delta \mathrm{S}_{\text {out }}\right]_{\mathrm{i}}^{\text {elast }}$ were calculated in the same defect configurations with the same atomic positions for an embedded cluster containing 54 atoms around the vacancy (and 445 atoms in the outer region), while using the atomic stress as defined by equation 19. The two sets of entropy changes are compared on figure 1 . The elastic corrections for the 445 atoms of the outer region are plotted as a function of their projected counterparts. Due to the residual symmetries of the saddle configuration, the number of distinct points of the plot is smaller than the number of atoms in the outer region. At the centre of the diagram, are plotted the corrections of minor amplitude observed for those sites which are far from the defect and associated with weak distortions; on the two wings, the corrections of larger amplitude are obtained for those atoms of the outer region which are closer to the boundary of the inner region defining the embedded cluster. Were the elastic correction perfect, then all the points would lie on the straight line. 
It is not the case, but it can be seen that the elastic correction is surprisingly good : the idea that the knowledge of the local deformation reproduces reasonnably the change in local entropy is thus physically sound. A detailed analysis of the correction on each site (graph not displayed here) shows that the elastic approximation is never in error concerning the expected sign of the correction.

The same calculation was done again while replacing the stress at the atomic level by an average including the stress on the site itself and an increasing number of neighbours $(12,18,42$ or 54$)$. The results for a stress averaged on 13 sites are displayed on figure $2:$ it is clearly seen that the scattering around the straight line is markedly broader: the same detailed analysis as above shows that now a noticeable fraction of the corrections proposed by the elastic approximation have a wrong sign when compared to their projected counterparts. When the stress is further averaged on more atoms, the corrections deviate still more strongly from the straight line : the larger the range of the coarse graining, the worse the results. As a conclusion, the most relevant information about the local disparities at the atomic scale, which is at work in the elastic correction, is progressively lost in the coarse grained averaging.

It is worth noticing that similar conclusions were reached in the study of point defect formation in amorphous silica: the formation energy of a Si vacancy depends on the Si site where it is formed. The distribution of formation energies is quantitatively explained by invoking the local pressure calculated on each $\mathrm{Si}$ atom prior to the vacancy formation. If this local pressure is replaced by the average pressure on the $\mathrm{SiO}_{4}$ tetrahedron (one $\mathrm{Si}$ atom and its 4 neighbouring $\mathrm{O}$ atoms), the tight correlation between formation energy and pressure vanishes and no explanation can be proposed to account for the distribution of formation energies [44-46]. Together with our results reported above, these findings should not be a surprise: after all, which better sensor of the surroundings can we think of than the atom itself immersed in the force field of its neighbours ?

The last point to examine is the presence of a strain gradient in the configurations to be studied. When looking at two first neighbour atoms ' $\mathrm{i}$ ' and ' $\mathrm{j}$ ', the strain gradient induces a change of their force constants $C_{i k}^{\alpha \beta}$ and $C_{j k}^{\alpha \beta}$ : the leading contribution to the production of eigen modes is provided by the diagonal terms. A close inspection of the force constant matrix in the distorted configuration shows that the diagonal coefficients $C_{i \mathrm{i}}^{\alpha \alpha}$ and $C_{\mathrm{j} j}^{\beta \beta}$ differ by no more than a fraction of a percent when passing from site a ' $\mathrm{i}$ ' to its first neighbour ' $\mathrm{j}$ ', provided both lie in the outer region. As a consequence, the change of the entropy is expected to be well within the errors implicitely introduced by the elastic approximation.

3.3.3 Application to defect studies. We will apply our approach to the formation of single-di-and-tri vacancy and of dumbbell interstitial as well as to the migration jump of these defects. The entropy difference between the perfect state and the imperfect one is made of two contributions. The contribution $\Delta \mathrm{S}_{\text {in }}$ of the inner region is given by:

$$
\Delta \mathrm{S}_{\text {in }} \square-\frac{\mathrm{k}}{2} \log \left(\Delta^{\prime} / \Delta^{\left(\mathrm{N}^{*}-\mathrm{N}_{\mathrm{d}}\right) / \mathrm{N}^{*}}\right)
$$

where $\mathrm{N}^{*}$ and $\mathrm{N}^{*}-\mathrm{N}_{\mathrm{d}}$ denote the number of atoms in the inner region of the perfect and imperfect state respectively $\left(\mathrm{N}_{\mathrm{d}}=-1,-2,-3\right.$ for a single-di-tri vacancy and +1 for a dumbbell). The contribution $\Delta S_{\text {out }}$ of the outer zone is given by equation 14 .

The pre-exponential factors $v_{0}$ of the jump frequencies are evaluated according to transition rate theory [47-48] for the inner region, the eigen modes of which must be calculated in the starting stable configuration and in the saddle 
configuration at the top of the energy barrier. The elastic correction is then applied to each site of the outer region after calculating the corresponding entropy change when going from the stable to the saddle configuration:

$$
v_{\mathrm{o}}=\frac{1}{2 \pi} \frac{\prod_{\mathrm{i}=1}^{3\left(\mathrm{~N}^{*}-\mathrm{N}_{\mathrm{d}}\right)} \omega_{\mathrm{i}}^{\text {stable }}}{\prod_{\mathrm{i}=1}^{3\left(\mathrm{~N}^{*}-\mathrm{N}_{\mathrm{d}}\right)-1} \omega_{\mathrm{i}}^{\text {saddle }}} \exp \left\{\left(\Delta \mathrm{S}_{\text {out }}^{\text {saddle }}-\Delta \mathrm{S}_{\text {out }}^{\text {stable })} / \mathrm{k}\right\}\right.
$$

For the case of defect clusters, the only jump studied here is non dissociative: the direction and the jumping partner are chosen in such a way that the structure of the defect cluster is restored (apart from a rotation) after the jump has been completed. In all cases, the atomic configurations used for the calculation of entropies are the results of a static relaxation involving all the atoms of the simulation cell $(\mathrm{N}=2048$ atoms); the relaxation is conducted under a constant external zero pressure as described in [3]. In the final stable or saddle states, the component of the forces are of the order of $10^{-6} \mathrm{eV} \mathrm{Angs}^{-1}$. The size of the inner region ranges from 55 to 791 . The entropies are also calculated in the supercell approach for $\mathrm{N}=256,500,864$, 1372 and 2048 as benchmark data.

\section{Introduction of the empirical potentials used in the study and results}

Before reviewing the four potentials used in this work, we draw the reader's attention on an important preliminary point which has been already mentionned by previous authors, namely the continuity of interactions at higher order [e.g. 15]. It is shown below that a discontinuity of third order can produce systematically faulty values of entropies. We illustrate this point with a simple potential of FinnisSinclair type in the subsequent section.

\subsection{Possible deleterious effects of a third order discontinuity on the entropy}

A simulation cell containing 864 atoms interacting through potential FS-PT3452.00 described in Appendix B is strained by a pure shear involving no volume change (strain $n^{\circ} 13$ in table 1 with an amplitude $\varepsilon \leq 10^{-3}$ ) and the calculated entropy change in units of $\mathrm{k}$ is plotted versus the square of the shear strain amplitude $\varepsilon^{2}$ in figure 3 . The expected quadratic behaviour, which is predicted by equation 14, is not obtained and a quasi linear variation is observed (top curve with a slope equal to $1 / 2$ ). It is however easy to check that a new calculation, switching the interactions to either FS-PT345-1.99, FS-PT456-2.00 or FS-PT456-1.99 cures entirely the anomaly and restores for the three lower curves in figure 3 the expected slope. The curves corresponding to the same cutoff radius 1.99 are superposed because the difference of the terminating polynomials does not play any role; the curve corresponding to the cutoff 2.00 is slightly higher since it establishes a vibrational connection between more distant neighbours.

Similar conclusions are reached for the formation entropies of a vacancy or a dumbbell: the potential FS-PT345-2.00 exhibits anomalously large values. The anomaly is cured by using the other versions of the potential. It is worth noticing that, in all cases, the energies are very close to one another (table 3 ).

(insert table 3 around here)

This faulty behaviour comes from the fact that the vibrational entropy is a function of the second derivatives of the interactions, and that a distortion of the lattice lets implicitly come into play higher order derivatives. In the present case, 
the origin of the flaw lies in the polynomial termination through which the interactions decay to zero: this polynomial has a discontinuity of its third derivative at $r_{\text {cut }}$ which was set equal to the fourth neighbour distance exactly. For the homogeneous shear strain under examination, some of the fourth neighbours enter or leave the interaction range: these displacements include unfortunately the discontinuity and induce an abrupt variation of the force constants. The flaw is cured, either by choosing a slightly smaller cutoff radius (1.99 isntead of 2) keeping the fourth neighbours out of reach, or by using a polynomial of higher order which changes the discontinuity at $r_{\text {cut }}$ from third to fourth order. Let us notice that we did not suppress the discontinuity of the third order derivative around $r_{r a c}$, but the latter distance is too far from any of the neighbour distances involved in the calculation of perfect or imperfect configurations to spoil the results.

It could obviously be argued that the choice of the cutoff distance in FSPT345-2.00 is very clumsy. Indeed, around single point defects, the strains of the embedding lattice are known with a good precision and the possible discontinuities of the potential (cutoff distance for the physical interactions, radius where an analytical form switches to another ...etc) can be carefully tuned in such a way that none of the neighbour shells will ever cross these points during the lattice deformation leading from the perfect reference state to the imperfect one. The choice is much more difficult, or even impossible, when dealing with situations involving large strains around complex defects like those produced by saddle configurations, interstitial or vacancy clusters, coherent interfaces with a large size misfit, grain-boundaries or local rearrangements during the collapse of a vacancy cluster into a vacancy loop ...etc. In all these cases, one can never be sure that some shell of neighbours will not cross a point where a third derivative of the potential is discontinuous. This is the reason why the analytical formulations of interactions for FS-ED-2.82 have been chosen with a damping term avoiding any discontinuity of any order.

It is worth noticing that this basic requirement of continuity was not always met in practice in previous investigations: although the basic interactions are evaluated with more and more sophisticated data including ab initio results, the quality of the final representation can be damaged by a functional expression which does not meet the requirement of continuity for high order derivatives. For instance, in [49] the embedding function is represented by cubic splines, the latter introducing as many discontinuities of the third order derivative as the number of mesh points; in [50] the embedding function is represented by two different expressions on the two sides of the equilibrium density $\rho_{\mathrm{o}}$ with a discontinuity of the third order derivative at $\rho_{0}$; in [51] the embedding function stems from an equation of state represented by two different expressions on the two sides of the equilibrium lattice parameter $\mathrm{a}_{\mathrm{o}}$; in this last case fortunately, the third order terms are identical on the two sides and the discontinuity shows up only at the fourth order derivative.

\subsection{Empirical potentials and related results}

The reason why we used several empirical potentials is not for the purpose of a quantitative comparison, which would have a minor interest: everybody knows that all of thems are basically wrong since none rests on a formulation of cohesion and atomic forces taking full account of the electronic structure. Their only virtue is to propose a simple phenomenological formulation of the interaction which allows a reasonable fit to some set of selected physical properties and experimental results. All the potentials examined below obey the general form of equations 17 and 18 
above, but their pairwise and density function terms are described by very different analytical expressions and are fitted to different physical properties.

Two potentials are of Finnis Sinclair type: i) the first one has a long interaction range with an exponential damping function, is fitted only to four physical properties of bulk copper and called hereafter FS-ED-2.82; ii) the second has a very short interaction range, is fitted to vibrational properties of copper surfaces [52] and called hereafter FS-Barreteau.

Two potentials are of EAM type: i) the first one is fitted to various properties of bulk copper including ab initio data [50]; we explain in Appendix B the way how it was modified to meet the requirement of continuity for its third derivative. This modified potential is called EAM-Mishin-mod; ii) the second one is explicitely fitted to elastic constants of second and third order [51] which have been experimentally determined for long [53] and called EAM-Milstein.

All the numerical details related to each potential are reported in Appendix B, including the resulting values of the elastic constants up to fourth order, the energetic and entropic parameters attached to the point defects under examination.

\subsection{Determination of entropic constants for the four potentials}

The entropic constants were determined using the procedure described in section 3.2. and are gathered in table 4:

* the first order entropic constant $\lambda_{1}$ has a comparable magnitude for the four potentials: it means that similar thermal expansion coefficients are predicted;

$*$ if $\lambda_{2}$ and $\lambda_{3}$ are different from one potential to the other, the difference $\lambda_{2}$ $\lambda_{3}$ has the same sign and the same order of magnitude, at least for the first three of them; EAM-Milstein is a special case and gives a larger result by one order of magnitude;

* unlike $\lambda_{2}$ and $\lambda_{3}$, the second order entropic constant $\lambda_{4}$ has a comparable order of magnitude, except for the potential EAM-Mishin-mod which yields a value twice smaller;

* the entropic constants $\lambda_{\mathrm{i}>4}$ corresponding to higher order terms are very different in magnitude and sign and do not exhibit any noticeable trend; fortunately, as will be verified below, they bring a hardly detectable contribution to the entropy change.

(insert table 4 around here)

\subsection{Effects of the elastic correction including shear strains}

We pass in review the effects of our elastic corrections on the configurations containing a defect enumerated in section 3.3.3. We compare the entropies obtained by the embedded cluster method in its original form (EC), with the elastic correction including pressure (ECp) and with our elastic correction including dilatation and shears (ECds). All the results are rated against those given by the supercell method (SC). The results obtained with the potentials FS-ED-2.82, FSBarreteau, EAM-Mishin-mod and EAM-Milstein will be displayed systematically in this order.

The form of equation 11 and 14 dictates the general trend to be expected: the magnitude and sign of the global entropic correction depends mainly on the magnitude and on the sign of the relaxation volume between the reference state and the imperfect one, since it provides the first order term. The additional effect of shear strains is of second order and shows up only in those configurations where shear stresses (and strains) are present with a noticeable magnitude. 
A last remark should be made here about the way with which the calculations are used, as well as about the relative importance of the ingredients entering the final results. The sites on which the elastic correction is applied are never in the close vicinity of the defect producing the strain; as a consequence, smaller strains than those used for determining the entropic constants are experienced, ranging typically from a few $10^{-3}$ to a few $10^{-6}$. However, we checked on preliminary calculations conducted on saddle configurations that the local strain tensor $\eta$ could not be extracted with an assumption of linear relationship between stress and strain. In other words the expression of the energy taking into account only the second order terms and corresponding to the first line in equation 8 is not sufficient and higher order terms are mandatory. As an illustration, we compare below in table 5 the entropic corrections evaluated in the stable and saddle configurations obtained with the potential FS-ED-2.82: i) with the only knowledge of the local pressure as proposed by equation 21 (or 22) and denoted by $\Delta S_{\text {out }}^{\text {stable }}$ (press) and $\Delta \mathrm{S}_{\text {out }}^{\text {saddle }}$ (press); ii) with the dilatation contributions $\Delta \mathrm{S}_{\text {out }}^{\text {stable }}$ (dilat), $\Delta \mathrm{S}_{\text {out }}^{\text {sadle }}$ (dilat) and shear contributions $\Delta S_{\text {out }}^{\text {stable }}$ (shear), $\Delta S_{\text {out }}^{\text {saddle }}$ (shear) as proposed by equation 14 of our approach.

For the latter, a preliminary remark should be made. The total entropy increase defined by equation 14 can be unambiguously partitionned into a first contribution $\Delta \mathrm{S}_{\mathrm{O} 1}$ coming from the unique first order term and a second contribution $\Delta \mathrm{S}_{\mathrm{O}>1}$ grouping higher order terms all together. Conversely its decomposition into contributions stemming from dilatation and shears respectively is somewhat arbitrary for two reasons: i) working with non linear elasticity, the definition of the pure dilatation part $\left\{\eta_{\mathrm{i}}\right\}^{\mathrm{d}}$ of the initial strain $\left\{\eta_{\mathrm{i}}\right\}$ according to $\left\{\eta_{i}\right\}=\left\{\eta_{i}\right\}^{d}+\left\{\eta_{i}\right\}^{s}$, defines a second term $\left\{\eta_{i}\right\}^{\text {s }}$ which is no longer a pure shear strain because it contains dilatation terms of second or higher order; ii) the fact that equation 14 contains non linear terms prevents us from decomposing formally the total entropy increase into two independent contributions corresponding to $\left\{\eta_{i}\right\}^{d}$ and $\left\{\eta_{i}\right\}^{s}$ respectively, because cross terms will unavoidably show up which mix

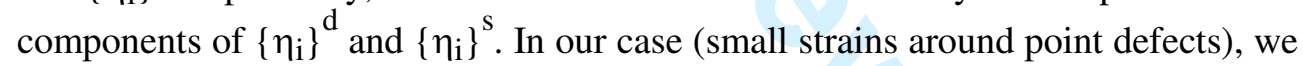
can however verify that these cross terms have a negligible contribution as follows. With equation 14, we can calculate independently from each other the entropic contributions $\left.\Delta \mathrm{S}($ dilat $)\right|_{\eta^{\mathrm{d}}}$ and $\left.\Delta \mathrm{S}($ shear $)\right|_{\eta^{\mathrm{s}}}$ due to a pure dilatation $\left\{\eta_{\mathrm{i}}\right\}^{\mathrm{d}}$ and an impure shear $\left\{\eta_{\mathrm{i}}\right\}^{\mathrm{S}}$ respectively. It is then checked readily that $\left.\Delta \mathrm{S}($ dilat $)\right|_{\eta^{\mathrm{d}}}$ and $\Delta \mathrm{S}$ (shear) $\left.\right|_{\eta \mathrm{s}}$ are nearly equal to $\Delta \mathrm{S}_{\mathrm{O} 1}$ and $\Delta \mathrm{S}_{\mathrm{O}>1}$ respectively, to better than one percent. This is the reason why the first order term in equation 14 will be considered, at least quantitatively, as the contribution of dilatation and displayed as such in subsequent table 5 .

The dilatational and shear contributions are calculated with stresses derived from an expression of the energy taking into account second order terms, second and third order terms and second, third and fourth order terms in equation 8, the corresponding results being denoted by $\mathrm{O}(2), \mathrm{O}(3)$ and $\mathrm{O}(4)$ respectively. The expressions used for the entropic correction are of the corresponding order.

(insert table 5 around here)

For the stable configuration, the final correction is negative, as expected, since the relaxation volume for vacancy formation is negative. The results $\mathrm{O}(2), \mathrm{O}(3)$ and $\mathrm{O}(4)$ are very close. The ECp and ECds approaches give the same result, which was a priori not obvious. In a continuous medium treated with isotropic elasticity 
the shear strains around a spherical hole have only tangential components. In the discrete medium made up of a FCC lattice, the pattern of internal stresses is more complicated: the dense rows $<110>$ running through the vacancy site are under tension while the others are compressed, thus leading to non zero radial shear components. But their influence on the final result is apparently very weak.

For the saddle configuration, a detailed inspection of the atomic sites shows that extracting the strains from stresses through formulas of increasing order enhances (algebraically) the local dilatation and decreases the weight of its negative contribution compared to that of shears. The final contribution due to dilatation remains however negative: indeed, the lattice parameter for the saddle point configuration is smaller than the parameter for the perfect lattice because the positive migration volume does not compensate entirely the negative relaxation volume observed during the vacancy formation. The contribution of the shears is conversely rather insensitive to the order of the approximation, which is probably due to the small strains experienced locally. As a result, the final entropic correction ECds is found negative with $\mathrm{O}(2)$ but it becomes positive with $\mathrm{O}(3)$; the additional contribution brought by $\mathrm{O}(4)$ terms is hardly detected. This correction ECds is to be compared to the ECp one, which is found negative. It illustrates the point already mentionned above in section 3.3.1: in [19], the change of pressure is assumed to stem only from a dilatation and it neglects the fact that shears bring also a quadratic contribution to pressure. In the particular case of a saddle point, the contribution of the shears becomes larger than the contribution of dilatation.

All our results displayed below and denoted by ECds are obtained after extracting the deformation through $\mathrm{O}(4)$ and evaluating the entropies with $\lambda_{\mathrm{i} \leq 4}$.

4.4.1. Formation entropies. The entropic corrections defined above are displayed in figure 4 for the dumbbell interstitial.

For the dumbbell, the EC values are much smaller than the SC ones. The first order term of the elastic correction stemming from the positive relaxation volume is positive and very large: the ECp values are higher than the EC ones by several $\mathrm{k}$ (figure 4). The ECds values are slightly higher than the ECp ones by one $\mathrm{k}$ at most. Although dissociated along a well defined direction, the dumbbell defect produces noticeable shear strains only in its close vicinity; the distortion of the lattice at larger distances is close to what would be produced by a center of dilatation of spherical symmetry, a feature which is reflected by three nearly equal eigen values of its elastic dipole tensor [20]: as a result, the contribution of the shears to the elastic is not very large.

For vacancy defects (graphs not shown for sake of space), the EC values are higher than the SC values. The relaxation volume is negative: as a consequence, the first order term of the elastic correction due to pressure or dilatation will bring a negative contribution. This correction makes the ECp and ECds results definitely closer to those obtained by supercell method, with some overshoot. For the single vacancy, the effect of shears is negligible which means that the vacancy behaves elastically as a contraction center of spherical symmetry: the ECds results are practically superposed upon the ECp ones. Conversely the poorer symmetry of diand tri-vacancy gives rise to a small but detectable contribution of shears which gives a further positive correction by a fraction of $\mathrm{k}$.

It is worth noticing that the amplitude of the elastic corrections are comparable for the four potentials, although the absolute values of the entropies (with or without the corrections) are noticeably different from one another.

In all cases, as expected, the second order correction due to shears decays more rapidly than the first order correction due to dilatation and is visible only for the smallest sizes of the inner region: it becomes hardly detectable as soon as $\mathrm{N}^{*}$ becomes larger than 500 .

(insert figures $4 \mathrm{a}$ to $4 \mathrm{~d}$ around here) 
4.4.2. Pre-exponential factor of jump frequencies. Saddle configurations are good candidates for involving large shear strains; but different situations are met according to the nature of the jumping defect. For sake of space we show only the migration results on figure 5 for the vacancy.

For the single vacancy, the migration volume is positive, and the relative position of the data points on the graph is the same as the one observed for the dumbbell formation. But now the additional contribution of the shears, for the smallest sizes of the inner region, is of the same order of magnitude as the contribution of pressure or dilatation alone.

For the di-vacancy and the tri-vacancy, the migration volumes are negative and the graphs look like those displayed for the formation of these defects. The effect of shears is more pronounced for the divacancy than for the tri-vacancy. As noticed in Appendix B for the saddle point configuration of the tri-vacancy jump, the jumping atom is close to the center of the tetrahedron made up by the three vacancies and the atom on their starting stable sites: this restores a more spherical symmetry for the radial displacements and decreases correspondingly the shears.

For the dumbbell, the migration volume is positive but very small. The same qualitative behaviour as the vacancy case is obtained but the overall change of the pre-exponential factor being less than $40 \%$ over the whole range swept by $1 / \mathrm{N}^{*}$, the graph does not look spectacular.

\section{(insert figures 5a to $5 \mathrm{~d}$ around here)}

\section{Conclusion}

The entropy change under a general strain can be expressed as a function of the strain components at all order with the help of entropic constants, which play for the entropy the same role as the elastic constants for the energy.

The use of non linear elasticity implies that a pressure change can be due to a dilatation or to a shear strain: it destroys the one-to-one correspondence between pressure and dilatation on one hand and shear stresses and shear strains on the other, which was the rule for linear elasticity. As a consequence the canonical variables to be chosen for expressing energy and entropy changes are the strain rather than the stress components.

Using the approximation of the embedded crystallite for the calculation of vibrational entropies attached to defect configurations, it has been confirmed that the elastic correction associated with the distorsion of the surrounding matrix is of noticeable importance.

Although the first order dilatational term brings in all cases a leading contribution, higher order term due to shear strains adds a noticeable improvement in all the situations of lowered symmetry, namely all those distorted configurations around defects or defect clusters in their stable or saddle configuration. The contribution of shear strains can be of the same order of magnitude as the dilatation one for saddle configurations.

It was further demonstrated that the main ingredient at work in the elastic approach, namely the local distortion, could be deduced in a meaningful way from stresses defined at atomic level.

These results are obtained with several empirical potentials, which differ from one another by various analytical formulations of their ingredients or by their interaction range. It suggests that the effect we enlightened in this study is general.

\section{Aknowledgements}


We thank E. Clouet, C. Marinica, B. Legrand, Y. Limoge and L. Proville for valuable discussions and suggestions and J.P. Poirier (Académie des Sciences) for drawing our attention on Brillouin's papers. 


\section{Appendix A: Calculation of $4^{\text {th }}$ and $5^{\text {th }}$ order elastic constants through lattice summations}

For the general form of the potential adopted here, the energy per atom ' $i$ ' is assumed to be expressed as:

$$
\mathrm{U}_{\mathrm{i}}=\frac{1}{2} \sum_{\mathrm{i} \neq \mathrm{j}} \mathrm{V}\left(\mathrm{r}_{\mathrm{ij}}\right)-\mathrm{F}\left(\rho_{\mathrm{i}}\right)
$$

where $\mathrm{F}\left(\rho_{\mathrm{i}}\right)$ is some (non linear) embedding function of the local density $\rho_{\mathrm{i}}$, the latter being a sum over interacting neighbors $\rho_{\mathrm{i}}=\sum_{\mathrm{i} \neq \mathrm{j}} \Phi\left(\mathrm{r}_{\mathrm{ij}}\right) . \mathrm{V}(\mathrm{r})$ and $\Phi(\mathrm{r})$ stand for two-body interactions.

The $\mathrm{n}^{\text {th }}$ order elastic constants are defined as the $\mathrm{n}^{\text {th }}$ derivatives of the energy with respect to the components of the applied strain and can be written synthetically under the form:

$$
\mathrm{C}_{\underbrace{}_{\mathrm{IJK}} \ldots}=\frac{1}{\Omega_{\mathrm{O}}} \frac{\partial^{\mathrm{n}} \mathrm{U}}{\partial \eta_{\mathrm{I}} \partial \eta_{\mathrm{J}} \partial \eta_{\mathrm{K}} \cdots}
$$

where $\Omega_{\mathrm{O}}$ stands for the atomic volume in the reference state and I, J, K are the Voigt indices. The derivatives $\frac{\partial}{\partial \eta_{\mathrm{I}}}$ are calculated via space derivatives $\frac{\partial}{\partial \mathrm{r}}$ of the functions $\mathrm{V}$ and $\Phi$, where $\mathrm{r}$ stands for the distance between some lattice site chosen as the origin and any other lattice site. If $\vec{r}_{O}=x_{1} \vec{a}+x_{2} \vec{b}+x_{3} \vec{c}$, where $(\vec{a}, \vec{b}, \vec{c})$ stands for some orthonormal set of basis vectors, is changed through the strain into $\overrightarrow{\mathrm{r}}=\mathrm{x}_{1} \overrightarrow{\mathrm{a}^{\prime}}+\mathrm{x}_{2} \overrightarrow{\mathrm{b}^{\prime}}+\mathrm{x}_{3} \overrightarrow{\mathrm{c}^{\prime}}$, then the change of distance can be expressed in a condensed form as $r^{2}-r_{O}^{2}=2 \sum_{I} X_{I} \eta_{I}$ where $X_{I}$ stands for the product of coordinates $\mathrm{x}_{\mathrm{k}} \mathrm{x}_{\mathrm{l}}$. From this expression stems the derivative $\mathrm{dr} / \mathrm{d} \eta_{\mathrm{I}}=\mathrm{X}_{\mathrm{I}} / \mathrm{r}$.

The calculation of the first derivatives of the energy $U$, which are nothing but the internal stresses resulting from the applied strain, is then straightforward:

$$
\Omega_{\mathrm{O}} \sigma_{\mathrm{I}}=\frac{\partial \mathrm{U}}{\partial \eta_{\mathrm{I}}}=\frac{1}{2} \sum_{\mathrm{r}} \frac{\mathrm{dV}}{\mathrm{dr}} \frac{\mathrm{dr}}{\mathrm{d} \eta_{\mathrm{I}}}-\frac{\mathrm{dF}}{\mathrm{d} \rho} \frac{\mathrm{d} \rho}{\mathrm{d} \eta_{\mathrm{I}}}=\frac{1}{2} \sum_{\mathrm{r}} \frac{\mathrm{dV}}{\mathrm{dr}} \frac{\mathrm{dr}}{\mathrm{d} \eta_{\mathrm{I}}}-\frac{\mathrm{dF}}{\mathrm{d} \rho} \sum_{\mathrm{r}} \frac{\mathrm{d} \Phi}{\mathrm{dr}} \frac{\mathrm{dr}}{\mathrm{d} \eta_{\mathrm{I}}}
$$

where the summations over ' $r$ ' are to be performed on all neighbours which interact with the central atom chosen as the origin. Hence the final formal expressions:

$$
\Omega_{\mathrm{O}} \sigma_{\mathrm{I}}=\frac{1}{2} \mathrm{SV}_{\mathrm{I}}-\frac{\mathrm{dF}}{\mathrm{d} \rho} \mathrm{S} \Phi_{\mathrm{I}}
$$

with the condensed notations

$$
\mathrm{SV}_{\mathrm{I}}=\sum_{\mathrm{r}} \frac{\mathrm{dV}}{\mathrm{dr}} \frac{\mathrm{dr}}{\mathrm{d} \eta_{\mathrm{I}}} \quad S \Phi_{\mathrm{I}}=\sum_{\mathrm{r}} \frac{\mathrm{d} \Phi}{\mathrm{dr}} \frac{\mathrm{dr}}{\mathrm{d} \eta_{\mathrm{I}}} .
$$

Denoting the products $X_{I} X_{J}$ by $X_{I J}, X_{I} X_{J} X_{K}$ by $X_{I J K} \ldots$ and extending the condensed notation to higher order derivatives of $\mathrm{V}$ and $\Phi$ according to : 


$$
\begin{aligned}
& \mathrm{Sf}_{\mathrm{IJ}}=\sum_{\mathrm{r}}\left(\frac{\mathrm{d}^{2} \mathrm{f}}{\mathrm{dr}^{2}}-\frac{1}{\mathrm{r}} \frac{\mathrm{df}}{\mathrm{dr}}\right) \frac{\mathrm{X}_{\mathrm{IJ}}}{\mathrm{r}^{2}} \\
& \mathrm{Sf}_{\mathrm{IJK}}=\sum_{\mathrm{r}}\left(\frac{\mathrm{d}^{3} \mathrm{f}}{\mathrm{dr}^{3}}-\frac{3}{\mathrm{r}} \frac{\mathrm{d}^{2} \mathrm{f}}{\mathrm{dr}^{2}}+\frac{3}{\mathrm{r}^{2}} \frac{\mathrm{df}}{\mathrm{dr}}\right) \frac{\mathrm{X}_{\text {IJK }}}{\mathrm{r}^{3}} \\
& \mathrm{Sf}_{\mathrm{IJKL}}=\sum_{\mathrm{r}}\left(\frac{\mathrm{d}^{4} \mathrm{f}}{\mathrm{dr}^{4}}-\frac{6}{\mathrm{r}} \frac{\mathrm{d}^{3} \mathrm{f}}{\mathrm{dr}^{3}}+\frac{15}{\mathrm{r}^{2}} \frac{\mathrm{d}^{2} \mathrm{f}}{\mathrm{dr}^{2}}-\frac{15}{\mathrm{r}^{3}} \frac{\mathrm{df}}{\mathrm{dr}}\right) \frac{\mathrm{X}_{\mathrm{IJKL}}}{\mathrm{r}^{4}} \\
& \mathrm{Sf}_{\mathrm{IJKLM}}=\sum_{\mathrm{r}}\left(\frac{\mathrm{d}^{5} \mathrm{f}}{\mathrm{dr}^{5}}-\frac{20}{\mathrm{r}} \frac{\mathrm{d}^{4} \mathrm{f}}{\mathrm{dr}^{4}}+\frac{45}{\mathrm{r}^{2}} \frac{\mathrm{d}^{3} \mathrm{f}}{\mathrm{dr}^{3}}-\frac{105}{\mathrm{r}^{3}} \frac{\mathrm{d}^{2} \mathrm{f}}{\mathrm{dr}^{2}}+\frac{105}{\mathrm{r}^{4}} \frac{\mathrm{df}}{\mathrm{dr}}\right) \frac{\mathrm{X}_{\text {IJKLM }}}{\mathrm{r}^{5}}
\end{aligned}
$$

where ' $\mathrm{f}$ ' stands for the $\mathrm{V}$ or $\Phi$ function. The expressions of the second, third, fourth and fifth order elastic constants are then easily deduced:

$$
\begin{aligned}
& \Omega_{\mathrm{O}} \mathrm{C}_{\mathrm{IJ}}=\frac{1}{2} \mathrm{SV} \mathrm{IJ}_{\mathrm{IJ}}-\frac{\mathrm{dF}}{\mathrm{d} \rho} \mathrm{S} \Phi_{\mathrm{IJ}}-\frac{\mathrm{d}^{2} \mathrm{~F}}{\mathrm{~d} \rho^{2}} \mathrm{~S} \Phi_{\mathrm{I}} \mathrm{S} \Phi_{\mathrm{J}} \\
& \Omega_{\bigcirc} C_{\mathrm{IJK}}=\frac{1}{2} \mathrm{SV}_{\mathrm{IJK}}-\frac{\mathrm{dF}}{\mathrm{d} \rho} \mathrm{S} \Phi_{\mathrm{IJK}} \\
& -\frac{\mathrm{d}^{2} \mathrm{~F}}{\mathrm{~d} \rho^{2}}\left[\mathrm{~S} \Phi_{\mathrm{I}} \mathrm{S} \Phi_{\mathrm{JK}}+\mathrm{S} \Phi_{\mathrm{J}} \mathrm{S} \Phi_{\mathrm{KI}}+\mathrm{S} \Phi_{\mathrm{K}} \mathrm{S} \Phi_{\mathrm{IJ}}\right] \\
& -\frac{\mathrm{d}^{3} \mathrm{~F}}{\mathrm{~d} \rho^{3}} S \Phi_{\mathrm{I}} S \Phi_{\mathrm{J}} S \Phi_{\mathrm{K}} \\
& \Omega_{\mathrm{O}} \mathrm{C}_{\mathrm{IJKL}}=\frac{1}{2} \mathrm{SV}_{\mathrm{IJKL}}-\frac{\mathrm{dF}}{\mathrm{d} \rho} \mathrm{S} \Phi_{\mathrm{IJKL}} \\
& -\frac{\mathrm{d}^{2} \mathrm{~F}}{\mathrm{~d} \rho^{2}}\left[\begin{array}{l}
\mathrm{S} \Phi_{\mathrm{I}} \mathrm{S} \Phi_{\mathrm{JKL}}+\mathrm{S} \Phi_{\mathrm{J}} \mathrm{S} \Phi_{\mathrm{KLI}}+\mathrm{S} \Phi_{\mathrm{K}} \mathrm{S} \Phi_{\mathrm{LIJ}}+\mathrm{S} \Phi_{\mathrm{L}} \mathrm{S} \Phi_{\mathrm{KL}}+\mathrm{S} \Phi_{\mathrm{IJK}} \mathrm{S} \Phi_{\mathrm{LI}}+\mathrm{S} \Phi_{\mathrm{KI}} \mathrm{S} \Phi_{\mathrm{JL}}
\end{array}\right] \\
& -\frac{d^{3} F}{d \rho^{3}}\left[\begin{array}{l}
S \Phi_{I J} S \Phi_{K} S \Phi_{L}+S \Phi_{I K} S \Phi_{J} S \Phi_{L}+S \Phi_{I L} S \Phi_{\mathrm{J}} S \Phi_{K}+ \\
S \Phi_{L}+S \Phi_{J L} S \Phi_{I} S \Phi_{K}+S \Phi_{K L} S \Phi_{I} S \Phi_{J}
\end{array}\right] \\
& -\frac{\mathrm{d}^{4} \mathrm{~F}}{\mathrm{~d} \rho^{4}} \mathrm{~S} \Phi_{\mathrm{I}} S \Phi_{\mathrm{J}} S \Phi_{\mathrm{K}} \mathrm{S} \Phi_{\mathrm{L}}
\end{aligned}
$$




$$
\Omega_{\mathrm{O}} \mathrm{C}_{\mathrm{IJKLM}}=\frac{1}{2} \mathrm{SV}_{\mathrm{IJKLM}}-\frac{\mathrm{dF}}{\mathrm{d} \rho} \mathrm{S} \Phi_{\mathrm{IJKLM}}
$$$$
-\frac{\mathrm{d}^{2} \mathrm{~F}}{\mathrm{~d} \rho^{2}}\left[\begin{array}{c}
\mathrm{S} \Phi_{\mathrm{IJK}} \mathrm{S} \Phi_{\mathrm{LM}}+\mathrm{S} \Phi_{\mathrm{IJL}} \mathrm{S} \Phi_{\mathrm{KM}}+\mathrm{S} \Phi_{\mathrm{IJM}} \mathrm{S} \Phi_{\mathrm{KL}}+\mathrm{S} \Phi_{\mathrm{IKL}} \mathrm{S} \Phi_{\mathrm{JM}}+\mathrm{S} \Phi_{\mathrm{IKM}} \mathrm{S} \Phi_{\mathrm{JL}} \\
+\mathrm{S} \Phi_{\mathrm{ILM}} \mathrm{S} \Phi_{\mathrm{JK}}+\mathrm{S} \Phi_{\mathrm{JKL}} \mathrm{S} \Phi_{\mathrm{IM}}+\mathrm{S} \Phi_{\mathrm{JKM}} \mathrm{S} \Phi_{\mathrm{IL}}+\mathrm{S} \Phi_{\mathrm{JLM}} \mathrm{S} \Phi_{\mathrm{IK}}+\mathrm{S} \Phi_{\mathrm{KLM}} \mathrm{S} \Phi_{\mathrm{IJ}} \\
+\mathrm{S} \Phi_{\mathrm{IJKL}} \mathrm{S} \Phi_{\mathrm{M}}+\mathrm{S} \Phi_{\mathrm{IJKM}} \mathrm{S} \Phi_{\mathrm{L}}+\mathrm{S} \Phi_{\mathrm{IJLM}} \mathrm{S} \Phi_{\mathrm{K}}+\mathrm{S} \Phi_{\mathrm{IKLM}} \mathrm{S} \Phi_{\mathrm{J}}+\mathrm{S} \Phi_{\mathrm{JKLM}} \mathrm{S} \Phi_{\mathrm{I}}
\end{array}\right]
$$$$
-\frac{\mathrm{d}^{3} \mathrm{~F}}{\mathrm{~d} \rho^{3}}\left[\begin{array}{l}
\mathrm{S} \Phi_{\mathrm{IJK}} \mathrm{S} \Phi_{\mathrm{L}} \mathrm{S} \Phi_{\mathrm{M}}+\mathrm{S} \Phi_{\mathrm{IJL}} \mathrm{S} \Phi_{\mathrm{K}} \mathrm{S} \Phi_{\mathrm{M}}+\mathrm{S} \Phi_{\mathrm{IJM}} \mathrm{S} \Phi_{\mathrm{K}} \mathrm{S} \Phi_{\mathrm{L}}+\mathrm{S} \Phi_{\mathrm{IKL}} \mathrm{S} \Phi_{\mathrm{J}} \mathrm{S} \Phi_{\mathrm{M}} \\
+\mathrm{S} \Phi_{\mathrm{IIM}} \mathrm{S} \Phi_{\mathrm{J}} \mathrm{S} \Phi_{\mathrm{L}}+\mathrm{S} \Phi_{\mathrm{ILM}} \mathrm{S} \Phi_{\mathrm{J}} \mathrm{S} \Phi_{\mathrm{K}}+\mathrm{S} \Phi_{\mathrm{JKL}} \mathrm{S} \Phi_{\mathrm{I}} \mathrm{S} \Phi_{\mathrm{M}} \mathrm{S} \Phi_{\mathrm{I}} \mathrm{S} \Phi_{\mathrm{K}}+\mathrm{S} \Phi_{\mathrm{IKL}} \mathrm{S} \Phi_{\mathrm{I}} \mathrm{S} \Phi_{\mathrm{L}} \mathrm{S} \Phi_{\mathrm{J}} \\
+\mathrm{S} \Phi_{\mathrm{IJ}} \mathrm{K} \Phi_{\mathrm{KL}} \mathrm{S} \Phi_{\mathrm{M}}+\mathrm{S} \Phi_{\mathrm{IJ}} \mathrm{S} \Phi_{\mathrm{KM}} \mathrm{S} \Phi_{\mathrm{L}}+\mathrm{S} \Phi_{\mathrm{IJ}} \mathrm{S} \Phi_{\mathrm{LM}} \mathrm{S} \Phi_{\mathrm{K}}+\mathrm{S} \Phi_{\mathrm{IK}} \mathrm{S} \Phi_{\mathrm{JL}} \mathrm{S} \Phi_{\mathrm{M}} \\
+\mathrm{S} \Phi_{\mathrm{IK}} \mathrm{S} \Phi_{\mathrm{JM}} \mathrm{S} \Phi_{\mathrm{L}}+\mathrm{S} \Phi_{\mathrm{IK}} \mathrm{S} \Phi_{\mathrm{LM}} \mathrm{S} \Phi_{\mathrm{J}}+\mathrm{S} \Phi_{\mathrm{IL}} \mathrm{S} \Phi_{\mathrm{JK}} \mathrm{S} \Phi_{\mathrm{M}}+\mathrm{S} \Phi_{\mathrm{IL}} \mathrm{S} \Phi_{\mathrm{JM}} \mathrm{S} \Phi_{\mathrm{K}} \\
+\mathrm{S} \Phi_{\mathrm{JK}} \mathrm{S} \Phi_{\mathrm{LM}} \mathrm{S} \Phi_{\mathrm{I}}+\mathrm{S} \Phi_{\mathrm{IM}} \mathrm{S} \Phi_{\mathrm{JL}} \mathrm{S} \Phi_{\mathrm{KK}} \mathrm{S} \Phi_{\mathrm{L}} \mathrm{S} \Phi_{\mathrm{I}}+\mathrm{S} \Phi_{\mathrm{IM}} \mathrm{S} \Phi_{\mathrm{JM}} \mathrm{S} \Phi_{\mathrm{JL}} \mathrm{S} \Phi_{\mathrm{K}}+\mathrm{S} \Phi_{\mathrm{I}} \mathrm{S} \Phi_{\mathrm{KL}} \mathrm{S} \Phi_{\mathrm{J}}
\end{array}\right]
$$$$
-\frac{d^{4} F}{d \rho^{4}}\left[\begin{array}{l}
S \Phi_{I J} S \Phi_{K} S \Phi_{L} S \Phi_{M}+S \Phi_{I K} S \Phi_{J} S \Phi_{L} S \Phi_{M}+S \Phi_{I L} S \Phi_{J} S \Phi_{K} S \Phi_{M} \\
+S \Phi_{I M} S \Phi_{J} S \Phi_{K} S \Phi_{L}+S \Phi_{J K} S \Phi_{I} S \Phi_{L} S \Phi_{M}+S \Phi_{J L} S \Phi_{I} S \Phi_{K} S \Phi_{M} \\
+S \Phi_{I} S \Phi_{K} S \Phi_{L}+S \Phi_{K L} S \Phi_{I} S \Phi_{J} S \Phi_{M}+S \Phi_{K M} S \Phi_{I} S \Phi_{J} S \Phi_{L} \\
+S \Phi_{L_{M}} S \Phi_{I} S \Phi_{J} S \Phi_{K}
\end{array}\right]
$$

$-\frac{d^{5} F}{d \rho^{5}} \quad S \Phi_{I} S \Phi_{J} S \Phi_{K} S \Phi_{L} S \Phi_{M}$

(A10) 


\section{Appendix B: Parameters of the interatomic interactions under examination for pure copper}

After a short description of the basic ingredients entering the definition of each potential, the resulting elastic constants (second, third and fourth order) are gathered in table B1, the formation and migration energies, volumes and entropies in table B2 at the end of the Appendix. Only those remarks specific for each potential are kept within the following subsections.

For all potentials, the tri-vacancy energy barrier has a double hump. In the starting configuration, the jumping atom is located on $(0,0,0)$ and the tri-vacancy on $(1,1,0)+(0,1,1)+(1,0,1)$; the jump exchanges the atom and the vacancy initially located on $(1,1,0)$. The saddle is found along $<111>$ at approximately $(0.25$, $0.25,0.25)$, the exact values depending on the potential. Then for all potentials, an intermediate metastable position shows up, the jumping atom being located at $(1 / 2,1 / 2,1 / 2)$. The depth of the well of metastability depends sharply on the range of the potentials; it corresponds to a shallow minimum for most potentials but one. The completion of the jump is performed when the atom crosses a second barrier of the same height in the $<11-1>$ direction.

We will deal only with interactions describing the total energy of the atomic assembly by:

$$
\mathrm{U}=\frac{1}{2} \sum_{\mathrm{i}} \sum_{\mathrm{i} \neq \mathrm{j}} \mathrm{V}\left(\mathrm{r}_{\mathrm{ij}}\right)-\sum_{\mathrm{i}} \mathrm{F}\left(\rho_{\mathrm{i}}\right)=\sum_{\mathrm{i}} \mathrm{U}_{\mathrm{i}}
$$

where $\mathrm{V}\left(\right.$ rij) stands for the pairwise part, $\mathrm{F}$ for the embedding function and $\rho_{\mathrm{i}}$ is a local density using a pairwise function $\Phi\left(\mathrm{r}_{\mathrm{ij}}\right)$ and given by :

$$
\rho_{\mathrm{i}}=\sum_{\mathrm{i} \neq \mathrm{j}} \Phi\left(\mathrm{r}_{\mathrm{ij}}\right) \text {. }
$$

This formulation allows to define an energy per atom denoted by $U_{i}$ and given by :

$$
\mathrm{U}_{\mathrm{i}}=\frac{1}{2} \sum_{\mathrm{j} \neq \mathrm{i}} \mathrm{V}\left(\mathrm{r}_{\mathrm{ij}}\right)-\mathrm{F}\left(\rho_{\mathrm{i}}\right)
$$

\section{B.1. Finnis-Sinclair type with various polynomial terminations and cutoff at finite distance (FS-PT-xxx)}

The interactions are modelled through exponentially decaying functions described by $V\left(r_{i j}\right)=\alpha \exp \left(-p r_{i j} / r_{o}\right), \quad \Phi\left(r_{i j}\right)=\exp \left(-2 q r_{i j} / r_{o}\right)$; the embedding function is given by $F\left(\rho_{i}\right)=\beta \rho_{i}^{1 / 2} \cdot r_{o}$ stands for the first neighbour distance in the perfect crystal at rest. The four adjustable parameters $\alpha, p, \beta, q$ are fitted on the cohesive energy $\mathrm{E}_{\mathrm{coh}}$, the bulk modulus $\mathrm{B}$, the lattice constant $\mathrm{a}_{\mathrm{o}}$ at rest (zero temperature and pressure), the unrelaxed formation energy of the vacancy $E_{f}$, while taking into account the first, second and third neighbour shells only.

Truncating the potential between third and fourth neighbours consists in replacing the exponential by a polynomial of fifth order, between some distance $r_{\text {rac }}$ arbitrarily chosen beyond the third neighbour distance $\left(r_{\text {rac }}=1.825 r_{0}\right)$ and the cutoff distance $\left(r_{\text {cut }}=2 r_{0}\right)$ of the form :

$$
\operatorname{Pol}(x)=a x^{3}+b x^{4}+c x^{5}
$$

where $x$ stands for $r-r_{\text {cut }}$. The polynomials used for $V(r)$ and $\Phi(r)$ will be called $\mathrm{P}(\mathrm{x})$ and $\mathrm{P}^{\prime}(\mathrm{x})$ respectively: the coefficients $\mathrm{a}, \mathrm{b}, \mathrm{c}$ for $\mathrm{P}(\mathrm{x})$ and $\mathrm{a}^{\prime}, \mathrm{b}^{\prime}, \mathrm{c}^{\prime}$ for $\mathrm{P}^{\prime}(\mathrm{x})$ are determined in such a way that the derivatives are continuous up to second order 
included at $r_{\text {rac }}$. By construction, the energy, the force and the curvature are zeroed at the cutoff distance $r_{\text {cut }}$. This potential fitted on copper $\left(E_{\mathrm{f}}=1.28 \mathrm{eV}, \mathrm{E}_{\mathrm{coh}}=3.54\right.$ $\mathrm{eV}, \mathrm{a}_{\mathrm{o}}=3.615$ Angs, $\mathrm{B}=0.864583 \mathrm{eVAngs}^{-3}$ ) is called FS-PT345-2.00 ('PT' for 'polynomial termination'; ' 345 ' for the exponents appearing in $\mathrm{P}(\mathrm{x})$ and $\mathrm{P}$ ' $(\mathrm{x}) ; 2.00$ for the value of $r_{\text {cut }} / r_{0}$ ). The coefficients are given by:

$$
\begin{array}{lll}
\alpha=0.1763567 \mathrm{eV} & \mathrm{p}=10.65757 \\
\beta=1.244282 \mathrm{eV} & \mathrm{q}=2.310711 \\
\mathrm{r}_{\mathrm{o}}=2.556191 \text { Angs } & \mathrm{r}_{\text {cut }}=2.00 \mathrm{r}_{\mathrm{o}}
\end{array}
$$

The polynomial Pol(x) can be replaced by a polynomial of higher order, namely $a x^{4}+b x^{5}+c x^{6}$, the coefficients of which are fitted to comply the same conditions at $r_{\text {rac }}$.

Three other variants of this potential are then defined combining the order of Pol(x) together with the cutoff distance, namely FS-PT345-1.99, FS-PT456-2.00 and FSPT456-1.99, which will be used in section 4.1.

\section{B.2. Finnis-Sinclair type with exponential damping and cutoff at finite distance (FS-ED-2.82)}

The expressions of the nude interactions used in section B.1 are damped by an exponential (hence the letters 'ED' in the name) which ensures that the functions and all their derivatives at any order vanish at the cutoff radius.

$$
\begin{aligned}
& \mathrm{V}\left(\mathrm{r}_{\mathrm{ij}}\right)=\alpha \exp \left\{-\mathrm{p} \mathrm{r}_{\mathrm{ij}} / \mathrm{r}_{\mathrm{o}}\right\} \exp \left\{\mathrm{a}_{\text {pair }} /\left(\mathrm{r}_{\mathrm{cut}}-\mathrm{r}_{\mathrm{ij}}\right)\right\} \\
& \Phi\left(\mathrm{r}_{\mathrm{ij}}\right)=\beta \exp \left\{-2 \mathrm{q} \mathrm{r}_{\mathrm{ij}} / \mathrm{r}_{\mathrm{o}}\right\} \exp \left\{\mathrm{a}_{\mathrm{phi}} /\left(\mathrm{r}_{\text {cut }}-\mathrm{r}_{\mathrm{ij}}\right)\right\}
\end{aligned}
$$

The cutoff distance was chosen in order to yield approximately the same values of $\mathrm{V}(\mathrm{r})$ and $\Phi(\mathrm{r})$ at first, second and third neighbour distances as those of the nude interactions in FS-PT456-2.00; the coefficients $a_{\text {pair }}$ and $a_{\text {phi }}$ of the damping functions are chosen in such a way as to keep a positive curvature all along the curve. Preliminary graphic examination shows that the seventh neighbour shell must be included, even if its numerical contribution is very small. The corresponding coefficients are listed hereafter:

$$
\begin{array}{lll}
\alpha=0.2356438 \mathrm{eV} & \mathrm{p}=10.26788 & \mathrm{a}_{\text {pair }}=-1 \text { Angs } \\
\beta=2.476014 \mathrm{eV}^{2} & \mathrm{q}=2.226221 & \mathrm{a}_{\mathrm{phi}}=-2 \text { Angs } \\
\mathrm{r}_{\mathrm{o}}=2.556191 \text { Angs } & \mathrm{r}_{\text {cut }}=2.81957444 \mathrm{r}_{\mathrm{o}} &
\end{array}
$$

\section{B.3. Finnis-Sinclair type with exponential damping and no cutoff (FS- Barreteau)}

This potential was originally designed to study vibrational and thermodynamical properties of $\mathrm{Cu}$ surfaces. The interaction range is kept small enough to match specific physical properties of copper surfaces, namely a correct sign and magnitude for the surface relaxation and high frequency (optical like) vibrational modes at surfaces clearly separated from those of the bulk by a gap of known magnitude : it retains mainly the contribution of first and second neighbours through the use of a damping function ' $\mathrm{fc}$ ' which is tuned to decay exponentially beyond a threshold radius $r_{c}$ slightly larger than the second neighbour distance: $\mathrm{fc}=\left[1+\exp \left\{\left(\mathrm{r}_{\mathrm{ij}}-\mathrm{r}_{\mathrm{c}}\right) / \delta\right\}\right]^{-1}$. The interactions are described by 
$\mathrm{V}\left(\mathrm{r}_{\mathrm{ij}}\right)=\alpha\left(\mathrm{r}_{\mathrm{o}} / \mathrm{r}_{\mathrm{ij}}\right)^{\mathrm{p}}$ and $\Phi\left(\mathrm{r}_{\mathrm{ij}}\right)=\exp \left(-2 \mathrm{q} \mathrm{r}_{\mathrm{ij}} / \mathrm{r}_{\mathrm{o}}\right)$; the embedding function is given by $\mathrm{F}\left(\rho_{\mathrm{i}}\right)=\beta \rho_{\mathrm{i}}^{2 / 3}$.

The damping function $\mathrm{fc}$ decreases very fast from 0.9997 at second neighbour distance to $4.110^{-10}$ at fourth neighbour distance. Although such a formulation requires in principle no cutoff radius, we used one set equal to $2.25 r_{o}$ between fourth and fifth neighbours in order to avoid an artificially null elastic constant $\mathrm{C}_{456}$ as mentioned in [28] and we checked that this modification did not bring any detectable change in the energies or entropies.

The corresponding coefficients are listed below:

$$
\begin{array}{llll}
\alpha=0.41112644734228 \text { eV } & \mathrm{p}=7.2055121109404 & \\
\beta=1.1021047271284 \text { eV } & \mathrm{q}=2.2205754785209 & \\
\mathrm{r}_{\mathrm{o}}=2.55265548 \text { Angs } & \mathrm{r}_{\mathrm{c}}=1.5748307 \mathrm{r}_{\mathrm{o}} & \delta=0.05
\end{array}
$$

For the migration of the tri-vacancy, the saddle point configurations raised difficulties; although the final state was relaxed correctly (residual force components as low as $10^{-5} \mathrm{eV} \mathrm{Angs}^{-1}$ ), several imaginary frequencies were found instead of one as expected.

The interaction range of this potential is so short that for the trivacancy jump, the local minimum in the intermediate metastable position becomes more stable than the stable starting configuration. The barrier top is somewhere around $(0.25,0.25$, 0.25 ). For the dumbbell, no reasonable saddle configuration could even be found with the drag method and more sophisticated algorithms should be used.

\section{B.4. EAM-Mishin-mod}

The original version of this potential has a pair term close to a Morse-function with a shallow minimum around the equilibrium distance $r_{0}$. The density function is monotously decreasing and the damping function for the two contributions is a rational fraction of fourth order. Additional short ranged terms are used to harden the repulsion at close approach distances:

The embedding function is represented by :

$$
\begin{aligned}
& F(\rho)=F_{o}+\frac{1}{2} F_{2}\left(\rho-\rho_{o}\right)^{2}+\sum_{n=1}^{4} q_{n}\left(\rho-\rho_{o}\right)^{n+2} \\
& F(\rho)=\frac{F_{o}+\frac{1}{2} F_{2}\left(\rho-\rho_{o}\right)^{2}+q_{1}\left(\rho-\rho_{o}\right)^{3}+Q_{1}\left(\rho-\rho_{o}\right)^{4}}{1+Q_{2}\left(\rho-\rho_{o}\right)^{3}}
\end{aligned}
$$

for $\rho<\rho_{O}$ and $\rho>\rho_{O}$ respectively. The density $\rho_{O}$ is further normalised to unity. Choosing for the embedding function different analytical formulas around the equilibrium value $\rho_{O}$ of the density leads to a discontinuity of its third order derivative. As a consequence, the calculation of entropies of a configuration containing a defect (vacancy, dumbbell, saddle configuration) might be altered by this ill-placed discontinuity because all these configurations involve a probing of $\mathrm{F}(\rho)$ on the two sides of the discontinuity. It was checked, however, that in the present case the consequences are not too deleterious: the differences are negligible for the energies and for the activation volumes; for the entropies, when noticeable, they remain small enough to be neglected.

The embedding function was replaced by a function having none of the drawbacks enlightened above. It is possible to reproduce the results already published with the original version of this potential through a simple fitting of the $\mathrm{N}$-body function by a fifth order polynomial for some neighbourhood of $\rho_{\mathrm{O}}$. For defect calculations (formation or migration), it can be checked that the range of $\rho$ 
values which is probed is rather narrow $0.5<\rho / \rho_{\mathrm{O}}<1.5$. As a consequence, a polynomial function of the form

$$
F(\rho)=q_{o}+\sum_{i=1}^{4} q_{i}\left(\rho-\rho_{o}\right)^{i+1}
$$

was fitted to the original values of the embedding function (available at http://cstwww.nrl.navy.mil/ccm6/ap/eam/index.html) in this interval and the coefficients were found to be :

$$
\begin{array}{lll}
\mathrm{q}_{\mathrm{o}}=-2.2823539 & \mathrm{q}_{1}=0.733148257 & \mathrm{q}_{2}=-0.763132346 \\
\mathrm{q}_{3}=0.124172653 & \mathrm{q}_{4}=0.683385904 &
\end{array}
$$

This modified analytical form of the embedding function was used to calculate the entropy change under strain. The basic quantities pertaining to defect formation and migration are gathered in table B2 and are very close to the ones published for the original version.

\section{B.5. EAM-Milstein}

The potential was primarily designed to account for second and third order elastic constants. For that purpose, the equation of state (EOS) is modified by replacing, in some range around the equilibrium lattice parameter $\mathrm{a}_{0}$, Rose's original expression by a seventh order polynomial. The values of all the coefficients can be found in the original paper, taking due account of the fact that $\gamma_{i}, \eta_{i}$ and A must be divided by 1.60219. The EOS function is continuous up to third order only.

However, no functional form for $F(\rho)$ was proposed in the original paper and we explain hereafter the way how we designed one. The values of $\rho$ and $F(\rho)$ were collected by varying the lattice parameter in the range $\left[0.8 \mathrm{a}_{0}: 1.5 \mathrm{a}_{\mathrm{o}}\right]$. The value of $\rho$ at $a_{o}$ is denoted by $\rho_{o}$ and used later on to normalize the original expression of the density. The resulting normalised values of $\rho$ belong to the interval $[0.3: 15]$. In this range, the embedding function $\mathrm{F}(\rho)$ can be represented fairly well by an expression of the form:

$$
\mathrm{F}(\rho)=\exp \left\{\mathrm{P}_{\mathrm{jp}-\mathrm{jp}+}\right\}-\exp \left\{\mathrm{Q}_{\mathrm{jq}-, \mathrm{jq}+}\right\}
$$

where $\mathrm{P}_{\mathrm{jp}-\mathrm{jp}+}$ and $\mathrm{P}_{\mathrm{jq}-\mathrm{jq}+}$ are polynomials containing powers of $\rho$ ranging from $\rho^{\mathrm{jp}-}$ to $\rho^{\mathrm{jp}+}$ and from $\rho^{\mathrm{jq}-}$ to $\rho^{\mathrm{jq}+}$ respectively, where jp- and jq-are negative, jp+ and jq+ are positive:

$$
\mathrm{P}=\sum_{\mathrm{jp}-}^{\mathrm{jp}+} \mathrm{p}(\mathrm{i}) \mathrm{r}^{\mathrm{i}} \quad \mathrm{Q}=\sum_{\mathrm{jq}-}^{\mathrm{jq}+} \mathrm{q}(\mathrm{i}) \mathrm{r}^{\mathrm{i}}
$$

The coefficients are then determined by a least square fit of the differences between the trial function and the target values. Numerous fits were tried and the best ones contained an equal number of positive and negative powers. The optimum was found for $\mathrm{P}_{-3: 3}$ and $\mathrm{Q}_{-3: 3}$ the coefficients of which are given below :

$$
\begin{array}{llll}
\mathrm{p}(-3)=-1.33414425293426 & 10^{-1} & \mathrm{q}(-3)=+1.56864346323235 & 10^{-2} \\
\mathrm{p}(-2)=+1.74078181863972 & 10^{-1} & \mathrm{q}(-2)=-1.04088096867450 & 10^{-1} \\
\mathrm{p}(-1)=-2.04037766897627 & \mathrm{q}(-1)=+1.14168394156699 & 10^{-1} \\
\mathrm{p}(0)=+2.46163195696694 & \mathrm{q}(0)=+8.57530003401110 & 10^{-1} \\
\mathrm{p}(1)=-8.58259043509680 & 10^{-3} & \mathrm{q}(1)=-8.33014047222441 & 10^{-2} \\
\mathrm{p}(2)=-4.87850283177439 & \mathrm{q}(2)=+1.47083060315825 & 10^{-3} \\
\mathrm{p}(3)=-2.81065015825138 & 10^{-1} & \mathrm{q}(3)=+4.97499743917570 & 10^{-5}
\end{array}
$$

In some interval denoted by $\left[\rho_{\min }: \rho_{\max }\right]$ around $\rho_{\mathrm{o}}$, the analytical representation is systematically too high (by $10^{-5}$ relative) and the minimum is not located exactly 
at $\rho_{\mathrm{o}}=1$. In this interval we subtracted a further exponential $-\exp (\mathrm{C})$ where $\mathrm{C}$ is also a polynomial containing powers of $\rho$ ranging from jc- to jc+, together with two more diverging terms at the bounds of the above interval :

$$
\mathrm{C}=\operatorname{cdiv}(1) /\left(\rho_{\min }-\rho\right)+\operatorname{cdiv}(2) /\left(\rho-\rho_{\max }\right)+\sum_{j c-}^{j \mathrm{c}+} \mathrm{c}(\mathrm{i}) \mathrm{r}^{\mathrm{i}}
$$

The coefficients where fitted to the differences between the target values and those attained by the approximation above, together with a further constraint on the first three derivatives at $\rho_{0}=1$ :

$$
\begin{array}{lllll}
c(-3)=-1.33414425293426 & 10^{-1} & \rho_{\min }=+0.86283783196531 \\
c(-2)=+1.74078181863972 & 10^{-1} & \rho_{\max }=+1.0607730618120 & \\
c(-1)=-2.04037766897627 & & \operatorname{cdiv}(1)=+0.14009094343182 & 10^{-1} \\
c(0)=+2.46163195696694 & & \operatorname{cdiv}(2)=+0.25927328768594 & 10^{-1} \\
c(1)=-8.58259043509680 & 10^{-3} & & \\
c(2)=-4.87850283177439 & & & \\
c(3)=-2.81065015825138 & 10^{-1} & &
\end{array}
$$

\section{B.6. Remarks}

(insert table B1, B2 here)

A few trends can be extracted after examining the above tables.

With regard to elastic constants:

* elastic constants of second and third order are reasonably similar; those produced by EAM-Milstein are by construction better than the others (except $\mathrm{C}_{123}$ which could not be tuned at will with the expression of the embedding function adopted above);

* for fourth order elastic constants, the last column of table B2 does not contain anything but a rough evaluation based on an approximate form of the repulsive interaction and on a value of $\mathrm{C}_{111}$ arbitrarily set equal to $12 \mathrm{eV} \mathrm{Angs}{ }^{-3}$ [53]. Surprisingly, the fourth order elastic constants produced by the three first potentials are definitely better than those produced by EAM-Milstein, although the latter was fitted with more refinement than the others to elastic properties.

With regard to parameters monitoring the formation of point defects:

* the potential FS-Barreteau is noticeably different from the others because of its very short range: the formation energies of vacancy defects are larger but the formation energy of the dumbbell is smaller; the reverse holds for the formation entropies;

* EAM-Milstein exhibits systematically higher formation entropies than all the other potentials for vacancy defects; but the reverse is true for the dumbbell.

With regard to parameters monitoring the migration of point defects:

* the pre-exponential factors do not differ from each other by more than one order of magnitude. 


\section{References}

[1] D. Frenkel and B. Smit in Understanding Molecular Simulation (Academic Press, San Diego, 2001).

[2] L. Zhao, R. Najafabadi, D.J. Srolovitz, Acta Mater. 442737 (1996).

[3] J.L. Bocquet, Mat. Sc. Forum 203-205 81 (2002).

[4] D.R. Mason, R.E. Rudd, A.P. Sutton, J. Physics-Condensed Matter 16 2679 (2004).

[5] G.D. Barrera, R.H. Tendler, Computer Physics Communications 105159 (1997).

[6] J.H. Harding, Rep. Prog. Phys. 531403 (1997).

[7] M. Athènes, Euro. Phys. J. B 38651 (2004).

[8] G. Adjanor, M. Athènes, F. Calvo, Euro. Phys. J. B 5347 (2006).

[9] N.W. Ashcroft and N.D. Mermin in Solid State Physics (Holt-Saunders International Edition, Philadelphia, 1976).

[10] M.J. Gillan, J.H. Harding, M. Leslie, Phys. C: Solid State Phys. 215465 (1988).

[11] R. LeSar, R. Najafabadi, D.J. Srolovitz, Phys. Rev. Letters 63624 (1989).

[12] J.M. Rickman, D.J. Srolovitz, Phil. Mag. A 671081 (1993).

[13] L. Zhao, R. Najafabadi, D.J. Srolovitz, Modelling Simul. Mater. Sci. Eng. 1 539 (1993).

[14] A.P. Sutton, Phil. Mag. 60147 (1989).

[15] A.P. Sutton, Phil. Trans. R. Soc. Lond. A 341233 (1992).

[16] R.D. Hatcher, R. Zeller, P.H. Dederichs, Phys. Rev. B 195083 (1979).

[17] M.J. Gillan , P.W.M. Jacobs, Phys. Rev. B 28759 (1983).

[18] S.B. De Biaggi, P.M. Decorte, A.M. Monti, Phys. Phys. Stat. Sol. (b) 19537 (1996).

[19] Y. Mishin, M.R. Sörensen, A.F. Voter, Phil. Mag. A 81, 2591 (2001).

[20] H. R. Schober, J. Phys. F: Metal Physics 71127 (1977).

[21] P.H. Dederichs, C. Lehmann, H.R. Schober, A. Scholz, R. Zeller, J. Nucl. Mat. 69-70 176 (1978).

[22] H. R. Schober, J. Nucl. Mat. 126220 (1984).

[23] J. R. Fernandez, A.M. Monti, Schober, Phys. Stat. Sol. (b) 179337 (1993).

[24] J.W. Martin, J. Phys. C: Solid State Phys. 82837 (1975).

[25] R. Brendel, J. de Physique : Colloque C8 (Suppt n¹1) 40 C8-189 (1979).

[26] C.S.G. Cousins, Proc. Phys. Soc. 91235 (1967).

[27] P.B. Ghate, J. Appl. Phys. 35337 (1964).

[28] S. Chantasiriwan, F. Milstein, Phys. Rev. B 5314080 (1996).

[29] L. Brillouin in Influence de la température sur l'élasticité d'un solide (Gauthiers-Villars Ed., Paris, 1940)

[30] C. Teodosiu in Elastic Models of Crystal Defects (Springer-Verlag, Berlin, 1982).

[31] M.B. Taylor, G.D. Barrera, N.L. Allan, T.H.K. Baron, Phys. Rev. B 5614380 (1997).

[32] M.B. Taylor, G.D. Barrera, N.L. Allan, T.H.K. Baron, W.C. Mackrodt, Computer Physics Communications 109135 (1998).

[33] S. Chantasiriwan, F. Milstein, Phys. Rev. B 585996 (1998).

[34] L.D. Landau, E.M. Lifshitz, in Theory of Elasticity (Pergamon Press, London, 1986).

[35] V. Vitek, T. Egami, Phys. Stat. Sol. (b) 144145 (1987).

[36] F. Berthier, B. Legrand, G. Tréglia, Acta Mater. 472705 (1999).

[37] F. Berthier, B. Legrand, G. Tréglia, Interface Science 855 (2000).

[38] S. Olivier, PhD Marseille, France (2004).

[39] D.H. Tsai, J. Chem. Phys. 701375 (1979).

[40] K.S. Cheung, S. Yip, J. Appl. Phys. 705688 (1991). 
[41] J.A. Zimmerman, E.B. Webb III, J.J. Hoyt, R.E. Jones, P.A. Klein, D.J. Bammann, Modelling Simul. Mater. Sci. Eng. 12 S319 (2004).

[42] J. Cormier, J.M. Rickman,T.J. Delph, J. Applied Phys. 8999 (2001).

[43] M. Zhou, Proc. R. Soc. London A459 2347 (2003).

[44] L. Martin-Samos, N. Richard, Y. Limoge, J.P. Crocombette, G. Roma, E. Englada, E. Artacho, EuroPhysics Letters 66880 (2004).

[45] L. Martin-Samos, N. Richard, Y. Limoge, J.P. Crocombette, G. Roma, E. Englada, E. Artacho, Phys. Rev. B71 014116 (2005).

[46] L. Martin-Samos, PhD Orsay, France (2004).

[47] G.H. Vineyard, J. Phys. Chem. Sol. 3121 (1957).

[48] G.H. Vineyard, J.A. Krumhansl, Phys. Rev. B 314929 (1985).

[49] Y. Mishin, D. Farkas, M.J. Mehl, D.A. Papaconstantopoulos, Phys. Rev. B 59 3393 (1999).

[50] Y. Mishin, D.A. Papaconstantopoulos, A.F. Voter, J.D. Kress, Phys. Rev B 63 224106 (2001).

[51] F. Milstein, S. Chantasiriwan, Phys. Rev. B 586006 (1998).

[52] C. Barreteau, F. Raouafi, M.C. Desjonquères, D. Spanjaard, Surface Science 507-510 748 (2002).

[53] Y. Hiki, A.V. Granato, Phys. Rev. 144411 (1966). 


\section{Table captions}

Table 1. Definition of the set of homogeneous strains applied to the simulation cell. Columns 1 to 6 give the independent components of the strains; column 7 gives the corresponding volume expansion; column 8 defines the numbers attached to the strains $\boldsymbol{\eta}$ defined by $\mathbf{J}$; column 9 defines the numbers attached to the strains at constant volume defined by $\mathbf{J} \mathrm{D}^{-1 / 3}$.

Table 2. Comparison of elastic constants of $2^{\text {nd }}$ to $4^{\text {th }}$ order obtained through lattice sums (column 2) and through a fitting to energy changes measured at finite strains (column 3) for potential FS-ED-2.82. The constants are multiplied by the atomic volume and the product $\Omega o C_{i j k l}$ is displayed (in $\mathrm{eV}$ ). The figures when identical in the two columns are bold faced.

Table 3. Formation energy (in eV) and entropy (in units of k) of vacancy and dumbbell defect obtained by Finnis-Sinclair potentials for copper with various terminations.

Table 4. Entropic constants of first, second and third order obtained with the four potentials investigated. The constants are expressed in units of $k$, per atom and per unit strain.

Table 5. Entropic corrections (in units of k) obtained for the stable and saddle configurations of a vacancy jump with potential FS-ED-2.82. The expression of the energy embodies second, third or fourth order terms and the corresponding contributions due to dilatation and shears are displayed separately; the last line is the correction based on the only knowledge of the local pressure.

Table B1. Elastic constants of second, third and fourth order obtained by lattice summations with the four potentials investigated (in $\mathrm{eV} \mathrm{Angs}^{-3}$ ). The last column contains the experimental values for second and third order constants and estimations of fourth order constants.

Table B2. Formation and migration energies, entropies, relaxation volumes for single vacancy (V1), divacancy (V2), trivacancy (V3) and $<100>$ dumbbell (I) defects in copper obtained by the four potentials investigated. Energies are expressed in $\mathrm{eV}$, relaxation and migration volumes in unit of atomic volume $\Omega_{\mathrm{o}}$, entropies in units of $\mathrm{k}$, pre-exponential factors $v_{\mathrm{o}}$ for migration in $\mathrm{s}^{-1}$. 


\begin{tabular}{|c|c|c|c|c|c|c|c|c|}
\hline $\mathbf{J}=$ & $\begin{array}{c}1+d \\
d_{6} \\
d_{5}\end{array}$ & & $\begin{array}{l}d_{6} \varepsilon \\
-d_{2} \varepsilon \\
d_{4} \varepsilon\end{array}$ & $\begin{array}{r}d \\
d \\
1+\end{array}$ & & $\operatorname{Det}[\mathbf{J}]=\mathrm{D}$ & $\eta=\mathbf{J}$ & $\eta=\mathbf{J} D^{-1 / 3}$ \\
\hline $\mathrm{d}_{1}$ & $\mathrm{~d}_{2}$ & $\mathrm{~d}_{3}$ & $\mathrm{~d}_{4}$ & $d_{5}$ & $\mathrm{~d}_{6}$ & $\Delta \mathrm{V} / \mathrm{V}$ & $\mathrm{n}^{\circ}$ & $\mathrm{n}^{\circ}$ \\
\hline 1 & 0 & 0 & 0 & 0 & 0 & $1+\varepsilon$ & 1 & 2 \\
\hline 1 & 1 & 0 & 0 & 0 & 0 & $1+2 \varepsilon+\varepsilon^{2}$ & 3 & 4 \\
\hline 1 & -1 & 0 & 0 & 0 & 0 & $1-\varepsilon^{2}$ & 5 & 6 \\
\hline 1 & 1 & 1 & 0 & 0 & 0 & $1+3 \varepsilon+3 \varepsilon^{2}+\varepsilon^{3}$ & 7 & \\
\hline 1 & 1 & -2 & 0 & 0 & 0 & $1-3 \varepsilon^{2}-2 \varepsilon^{3}$ & 8 & 9 \\
\hline 0 & 0 & 0 & 1 & 0 & 0 & $1-\varepsilon^{2}$ & & 10 \\
\hline 0 & 0 & 0 & 1 & 1 & 0 & $1-2 \varepsilon^{2}$ & & 11 \\
\hline 0 & 0 & 0 & 1 & -1 & 0 & $1-2 \varepsilon^{2}$ & & 12 \\
\hline 0 & 0 & 0 & 1 & 1 & 1 & $1-3 \varepsilon^{2}+2 \varepsilon^{3}$ & & 13 \\
\hline 0 & 0 & 0 & 1 & -1 & 1 & $1-3 \varepsilon^{2}-2 \varepsilon^{3}$ & & 14 \\
\hline 0 & 1 & -1 & 1 & 0 & 0 & $1-2 \varepsilon^{2}$ & & 15 \\
\hline 0 & 1 & -1 & -1 & 0 & 0 & $1-2 \varepsilon^{2}$ & & 16 \\
\hline 0 & 1 & -1 & 0 & 0 & 1 & $1-2 \varepsilon^{2}+\varepsilon^{3}$ & & 17 \\
\hline 0 & 1 & -1 & 0 & 0 & -1 & $1-2 \varepsilon^{2}+\varepsilon^{3}$ & & 18 \\
\hline 0 & 1 & -1 & 1 & 1 & 0 & $1-3 \varepsilon^{2}-\varepsilon^{3}$ & & 19 \\
\hline 0 & 1 & -1 & 1 & -1 & & $1-3 \varepsilon^{2}-\varepsilon^{3}$ & & 20 \\
\hline 0 & 1 & -1 & 1 & 1 & 1 & $1-4 \varepsilon^{2}+2 \varepsilon^{3}$ & & 21 \\
\hline 0 & 1 & -1 & 1 & 1 & -1 & $1-4 \varepsilon^{2}+2 \varepsilon^{3}$ & & 22 \\
\hline 0 & 1 & -1 & 1 & -1 & 1 & $1-4 \varepsilon^{2}+2 \varepsilon^{3}$ & & 23 \\
\hline 2 & -1 & -1 & 1 & 1 & 1 & $1-6 \varepsilon^{2}+4 \varepsilon^{3}$ & & 24 \\
\hline 2 & -1 & -1 & -1 & 1 & 1 & $1-6 \varepsilon^{2}+4 \varepsilon^{3}$ & & 25 \\
\hline 2 & -1 & -1 & 1 & -1 & 1 & $1-6 \varepsilon^{2}+4 \varepsilon^{3}$ & & 26 \\
\hline 0 & 0 & 0 & 1 & 1 & -1 & $1-3 \varepsilon^{2}-2 \varepsilon^{3}$ & 27 & \\
\hline 1 & 0 & 0 & 1 & 0 & 0 & $1+\varepsilon-\varepsilon^{2}-\varepsilon^{3}$ & 29 & 28 \\
\hline 1 & 0 & 0 & 0 & 1 & 1 & $1+\varepsilon-2 \varepsilon^{2}$ & 31 & 30 \\
\hline-1 & 0 & 0 & 1 & 0 & 0 & $1-\varepsilon-\varepsilon^{2}+\varepsilon^{3}$ & 33 & 32 \\
\hline-1 & 0 & 0 & 0 & -1 & 1 & $1-\varepsilon-2 \varepsilon^{2}$ & 35 & 34 \\
\hline
\end{tabular}

Table 1 


\begin{tabular}{|l|l|l|}
\hline Elastic constants & $\Omega_{\mathrm{O}} \mathrm{C}_{\mathrm{ijkl}}$ (finite strains) & $\Omega_{\mathrm{O}} \mathrm{C}_{\mathrm{ijkl}}$ (lattice sums) \\
\hline $\mathrm{C}_{11}$ & 6.2475235685114 & 6.2474252388986 \\
\hline $\mathrm{C}_{12}$ & 9.0492167231813 & 9.0491240900153 \\
\hline $\mathrm{C}_{44}$ & 2.8251017423355 & 2.8250716966494 \\
\hline & & \\
\hline $\mathrm{C}_{111}$ & -17.828969522483 & -17.823955949629 \\
\hline $\mathrm{C}_{112}$ & -31.312129221365 & -31.290734067052 \\
\hline $\mathrm{C}_{123}$ & -3.6656731068476 & -3.6340037491056 \\
\hline $\mathrm{C}_{144}$ & -1.3845306831346 & -1.3830762314058 \\
\hline $\mathrm{C}_{155}$ & -27.327019695211 & -27.278818705580 \\
\hline $\mathrm{C}_{456}$ & 2.0145253546836 & 2.0085900124411 \\
\hline $\mathrm{C}_{1111}$ & & \\
\hline $\mathrm{C}_{1112}$ & 38.235065289803 & 36.400906868595 \\
\hline $\mathrm{C}_{1122}$ & 72.858873634422 & 76.454546757452 \\
\hline $\mathrm{C}_{1123}$ & 101.09643557587 & 112.67670620445 \\
\hline $\mathrm{C}_{1144}$ & 19.837733029522 & 3.7343049428951 \\
\hline $\mathrm{C}_{1155}$ & -0.26215922978722 & 0.41667730232131 \\
\hline $\mathrm{C}_{1244}$ & 105.95814600150 & 106.62818222498 \\
\hline $\mathrm{C}_{1356}$ & 2.5542637637227 & 3.5628063323601 \\
\hline $\mathrm{C}_{1456}$ & 212.68620161031 & 214.00605228198 \\
\hline $\mathrm{C}_{4444}$ & -18.099248594098 & -4.7288084307735 \\
\hline $\mathrm{C}_{4455}$ & 17.327311252021 & 17.455712085607 \\
\hline & 0.063896262016610 & 0.024534599878828 \\
\hline & & \\
\hline & & \\
\hline
\end{tabular}

Table 2 


\begin{tabular}{ccccc}
\hline & FS-PT345-2.00 & FS-PT345-1.99 & FS-PT456-2.00 & FS-PT456-1.99 \\
\hline $\mathrm{E}_{\mathrm{F}}^{\mathrm{V}}$ & 1.249 & 1.250 & 1.2496 & 1.2498 \\
$\mathrm{~S}_{\mathrm{F}}^{\mathrm{V}} / \mathrm{k}$ & 4.71 & 1.55 & 1.60 & 1.39 \\
$\mathrm{E}_{\mathrm{F}}^{\mathrm{I}}$ & 2.959 & 2.980 & 2.997 & 2.954 \\
$\mathrm{~S}_{\mathrm{F}}^{\mathrm{I}} / \mathrm{k}$ & 18.95 & 12.72 & 12.25 & 11.33 \\
\hline
\end{tabular}

Table 3 


\begin{tabular}{cccccc}
\hline \multicolumn{2}{c}{ Entropic constants } & $\begin{array}{c}\text { FS } \\
\text { ED-2.82 }\end{array}$ & $\begin{array}{c}\text { FS } \\
\text { Barreteau }\end{array}$ & $\begin{array}{c}\text { EAM } \\
\text { Mishin- } \\
\text { mod }\end{array}$ & $\begin{array}{c}\text { EAM } \\
\text { Milstein }\end{array}$ \\
full name & short name & & & & \\
$\lambda_{1}$ & $\lambda_{1}$ & +6.570 & +6.199 & +5.365 & +7.054 \\
& & & & & \\
$\lambda_{11}$ & $\lambda_{2}$ & +3.982 & +2.77 & -3.99 & -23.00 \\
$\lambda_{12}$ & $\lambda_{3}$ & -1.676 & +0.42 & -9.67 & -64.93 \\
$\lambda_{44}$ & $\lambda_{4}$ & +13.19 & +13.70 & +7.35 & +14.50 \\
$\lambda_{111}$ & $\lambda_{5}$ & +0.85 & -143 & +120 & +631 \\
$\lambda_{112}$ & $\lambda_{6}$ & +9.4 & -62 & +191 & +1743 \\
$\lambda_{123}$ & $\lambda_{7}$ & +50 & -18 & +7 & +3025 \\
$\lambda_{144}$ & $\lambda_{8}$ & +42 & -0.011 & +20 & +96 \\
$\lambda_{155}$ & $\lambda_{9}$ & -31 & -99 & +177 & +165 \\
$\lambda_{456}$ & $\lambda_{10}$ & -0.347 & -88 & -7.3 & -92 \\
\hline
\end{tabular}

Table 4 


\begin{tabular}{|c|c|c|c|c|}
\hline Correction & \multicolumn{2}{|c|}{ stable configuration } & \multicolumn{2}{|c|}{ saddle configuration } \\
\hline \multirow{2}{*}{$\begin{array}{c}\text { with full } \\
\text { strain tensor }\end{array}$} & $\Delta \mathrm{S}_{\text {out }}^{\text {stable }}$ (dilat) & $\Delta S_{\text {out }}^{\text {stable }}($ shear $)$ & $\Delta S_{\text {out }}^{\text {saddle }}($ dilat $)$ & $\Delta S_{\text {out }}^{\text {saddle }}$ (shear) \\
\hline & $\mathrm{k}$ & $\mathrm{k}$ & $\overline{\mathrm{k}}$ & $\mathrm{k}$ \\
\hline $\mathrm{O}(2)$ & -0.615 & +0.00882 & -0.398 & +0.169 \\
\hline $\mathrm{O}(3)$ & -0.601 & +0.00886 & -0.115 & +0.180 \\
\hline $\mathrm{O}(4)$ & -0.601 & +0.00886 & -0.122 & +0.176 \\
\hline with local & \multicolumn{2}{|c|}{$\Delta \mathrm{S}_{\text {out }}^{\text {stable }}$ (press) } & \multicolumn{2}{|c|}{$\Delta \mathrm{S}_{\text {out }}^{\text {saddle }}$ (press) } \\
\hline pressure & \multicolumn{2}{|c|}{$\begin{array}{c}\mathrm{k} \\
\end{array}$} & \multicolumn{2}{|c|}{$\begin{array}{c}\mathrm{k} \\
\end{array}$} \\
\hline 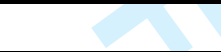 & \multicolumn{2}{|c|}{-0.605} & \multicolumn{2}{|c|}{-0.201} \\
\hline
\end{tabular}

Table 5 


\begin{tabular}{|c|c|c|c|c|c|}
\hline & $\begin{array}{c}\text { FS } \\
\text { ED-2.82 }\end{array}$ & $\begin{array}{c}\text { FS } \\
\text { Barreteau }\end{array}$ & $\begin{array}{c}\text { EAM } \\
\text { Mishin } \\
\text { modified }\end{array}$ & $\begin{array}{c}\text { EAM } \\
\text { Milstein }\end{array}$ & Experiments \\
\hline $\mathrm{C}_{11}$ & +1.058 & +1.113 & +1.084 & +1.104 & +1.0997 \\
\hline $\mathrm{C}_{12}$ & +0.766 & +0.785 & +0.789 & +0.785 & +0.7798 \\
\hline $\mathrm{C}_{44}$ & +0.478 & +0.517 & +0.476 & +0.510 & +0.5104 \\
\hline $\mathrm{C}_{111}$ & -9.055 & -8.752 & -7.983 & -11.024 & -12.483 \\
\hline $\mathrm{C}_{112}$ & -5.299 & -5.197 & -4.128 & -6.157 & -7.6146 \\
\hline $\mathrm{C}_{123}$ & -0.3077 & -0.349 & +0.477 & -1.663 & -3.1207 \\
\hline $\mathrm{C}_{144}$ & -0.234 & -0.233 & -0.283 & -0.838 & -0.8239 \\
\hline $\mathrm{C}_{155}$ & -4.619 & -4.557 & -4.256 & -4.400 & -4.4002 \\
\hline $\mathrm{C}_{456}$ & +0.170 & +0.098 & +0.113 & +0.160 & +0.1560 \\
\hline $\mathrm{C}_{1111}$ & +73.970 & +75.100 & +47.554 & +10.155 & $\approx+[84: 168]$ \\
\hline $\mathrm{C}_{1112}$ & +38.841 & +31.394 & +17.342 & +16.637 & $\approx+[42: 84]$ \\
\hline $\mathrm{C}_{1122}$ & +38.162 & +34.342 & +17.084 & +12.868 & $\approx+[42: 84]$ \\
\hline $\mathrm{C}_{1123}$ & +0.632 & -1.944 & -7.736 & -54.352 & $\approx 0$ \\
\hline $\mathrm{C}_{1144}$ & +0.141 & -2.698 & -0.203 & -6.280 & $\approx 0$ \\
\hline $\mathrm{C}_{1155}$ & +36.113 & +28.149 & +25.920 & +44.207 & $\approx+[42: 84]$ \\
\hline $\mathrm{C}_{1244}$ & +0.603 & -2.204 & +0.2621 & +10.784 & $\approx 0$ \\
\hline $\mathrm{C}_{1266}$ & +36.240 & +32.341 & +24.875 & +54.976 & $\approx+[42: 84]$ \\
\hline $\mathrm{C}_{1456}$ & -0.400 & -3.152 & +0.312 & -9.239 & $\approx 0$ \\
\hline $\mathrm{C}_{4444}$ & +35.472 & +31.140 & +25.017 & +25.188 & $\approx+[42: 84]$ \\
\hline $\mathrm{C}_{4455}$ & +0.012 & -2.817 & +0.666 & -5.809 & $\approx 0$ \\
\hline
\end{tabular}

Table B1 


\begin{tabular}{|c|c|c|c|c|}
\hline & $\begin{array}{c}\text { FS } \\
\text { ED-2.82 }\end{array}$ & $\begin{array}{c}\text { FS } \\
\text { Barreteau }\end{array}$ & $\begin{array}{c}\text { EAM } \\
\text { Mishin } \\
\text { mod }\end{array}$ & $\begin{array}{c}\text { EAM } \\
\text { Milstein }\end{array}$ \\
\hline $\mathrm{E}_{\mathrm{F}}^{\mathrm{V}}$ & 1.203 & 1.513 & 1.273 & 1.305 \\
\hline $\mathrm{S}_{\mathrm{F}}^{\mathrm{V}} / \mathrm{k}$ & 1.387 & 1.232 & 1.408 & 2.355 \\
\hline$\Delta \mathrm{V}_{\mathrm{F}}^{\mathrm{V}} / \Omega_{\mathrm{O}}$ & +0.742 & +0.763 & +0.700 & +0.762 \\
\hline $\mathrm{E}_{\mathrm{M}}^{\mathrm{V}}$ & 0.672 & 0.547 & 0.689 & 0.682 \\
\hline$\Delta \mathrm{V}_{\mathrm{M}}^{\mathrm{V}} / \Omega_{\mathrm{O}}$ & +0.155 & +0.0696 & +0.106 & +0.101 \\
\hline $10^{-13} v_{\mathrm{O}}^{\mathrm{V}}$ & 1.372 & 0.4288 & 0.7 .665 & 0.6109 \\
\hline $\mathrm{E}_{\mathrm{F}}^{\mathrm{V} 2}$ & 2.270 & 2.881 & 2.401 & 2.440 \\
\hline $\mathrm{S}_{\mathrm{F}}^{\mathrm{V} 2} / \mathrm{k}$ & 2.768 & 2.371 & 2.734 & 4.577 \\
\hline$\Delta \mathrm{V}_{\mathrm{F}}^{\mathrm{V} 2} / \Omega_{\mathrm{O}}$ & +1.479 & +1.541 & +1.385 & +1.543 \\
\hline $\mathrm{E}_{\mathrm{M}}^{\mathrm{V} 2}$ & 0.358 & 0.278 & 0.364 & 0.442 \\
\hline$\Delta \mathrm{V}_{\mathrm{M}}^{\mathrm{V} 2} / \Omega_{\mathrm{O}}$ & -0.099 & -0.223 & -0.132 & -0.221 \\
\hline $10^{-13} v_{\mathrm{O}}^{\mathrm{V} 2}$ & 4.185 & 2.158 & 1.298 & 1.738 \\
\hline $\mathrm{E}_{\mathrm{F}}^{\mathrm{V} 3}$ & 3.198 & 4.273 & 3.379 & 3.392 \\
\hline $\mathrm{S}_{\mathrm{F}}^{\mathrm{V} 3} / \mathrm{k}$ & 4.071 & 3.283 & 3.925 & 6.392 \\
\hline$\Delta \mathrm{V}_{\mathrm{F}}^{\mathrm{V} 3} / \Omega_{\mathrm{O}}$ & +2.223 & +2.371 & +2.086 & +2.383 \\
\hline $\mathrm{E}_{\mathrm{M}}^{\mathrm{V} 3}$ & 0.061 & 0.0915 & 0.0643 & 0.231 \\
\hline$\Delta \mathrm{V}_{\mathrm{M}}^{\mathrm{V} 3} / \Omega_{\mathrm{O}}$ & -0.368 & -0.560 & -0.364 & -0.708 \\
\hline $10^{-13} v_{\mathrm{O}}^{\mathrm{V} 3}$ & 0.7352 & $?$ & 0.7409 & 1.371 \\
\hline $\mathrm{E}_{\mathrm{F}}^{\mathrm{I}}$ & 3.034 & 2.616 & 3.073 & 3.188 \\
\hline $\mathrm{S}_{\mathrm{F}}^{\mathrm{I}} / \mathrm{k}$ & 10.245 & 11.017 & 7.428 & 9.998 \\
\hline$\Delta \mathrm{V}_{\mathrm{R}}^{\mathrm{I}} / \Omega_{\mathrm{O}}$ & +0.997 & +0.770 & +0.825 & +1.225 \\
\hline $\mathrm{E}_{\mathrm{M}}^{\mathrm{I}}$ & 0.084 & $?$ & 0.0974 & 0.0741 \\
\hline$\Delta \mathrm{V}_{\mathrm{M}}^{\mathrm{I}} / \Omega_{\mathrm{O}}$ & +0.0287 & $?$ & +0.0386 & +0.0213 \\
\hline $10^{-13} v_{\mathrm{O}}^{\mathrm{I}}$ & 0.1501 & $?$ & 0.1982 & 0.04712 \\
\hline
\end{tabular}

Table B2 


\section{Figure captions}

Figure 1. Comparison between the projected entropies (abscissas) and the entropies calculated with the elastic correction (ordinates): on every site, the elastic correction rests on a local strain deduced from the atomic stresses on this site.

Figure 2. Comparison between the projected entropies (abscissas) and the entropies calculated with the elastic correction (ordinates): on every site, the elastic correction rests on a local strain deduced from the atomic stresses coarse-grained over 13 atoms.

Figure 3. Entropy change (in units of k) of a cubic box containing 864 atoms under a uniform shear stress of amplitude $\varepsilon$ in a $\log -\log$ plot versus $\varepsilon^{2}$ for different potentials. From top to bottom: the empty circles ( $($ ) stand for potential FS-PT3452.00, the empty squares ( $\square$ ) stand for potential FS-PT456-2.00, the empty diamonds $(\diamond)$ stand for potential FS-PT345-1.99, the multiply signs $(\times)$ stand for potential FS-PT456-1.99. Two straight lines of slope 1/2 (top) and 1 (bottom) are displayed for comparison.

Figure 4. Formation entropy of dumbbell (in units of k) versus the inverse number $\mathrm{N}^{*}$ of atoms in the inner region. (a) FS-ED-2.82; (b) FS-Barreteau; (c) EAMMishin-mod; (d) EAM-Milstein. The 'plus' symbols (+) stand for the embedded crystallite results; the 'multiply' symbols $(x)$ for the elastic correction including pressure; the empty squares $(\square)$ for the elastic correction including dilatation and shears; the filled triangles $(\boldsymbol{\Lambda})$ for the supercell results.

Figure 5. Pre-exponential factor $v_{0}$ for the vacancy jump (in $\mathrm{s}^{-1}$ ) versus the inverse number $\mathrm{N}^{*}$ of atoms in the inner region. (a) FS-ED-2.82; (b) FS-Barreteau; (c) EAM-Mishin-mod; (d) EAM-Milstein. Same meaning of symbols as in figure 4. 


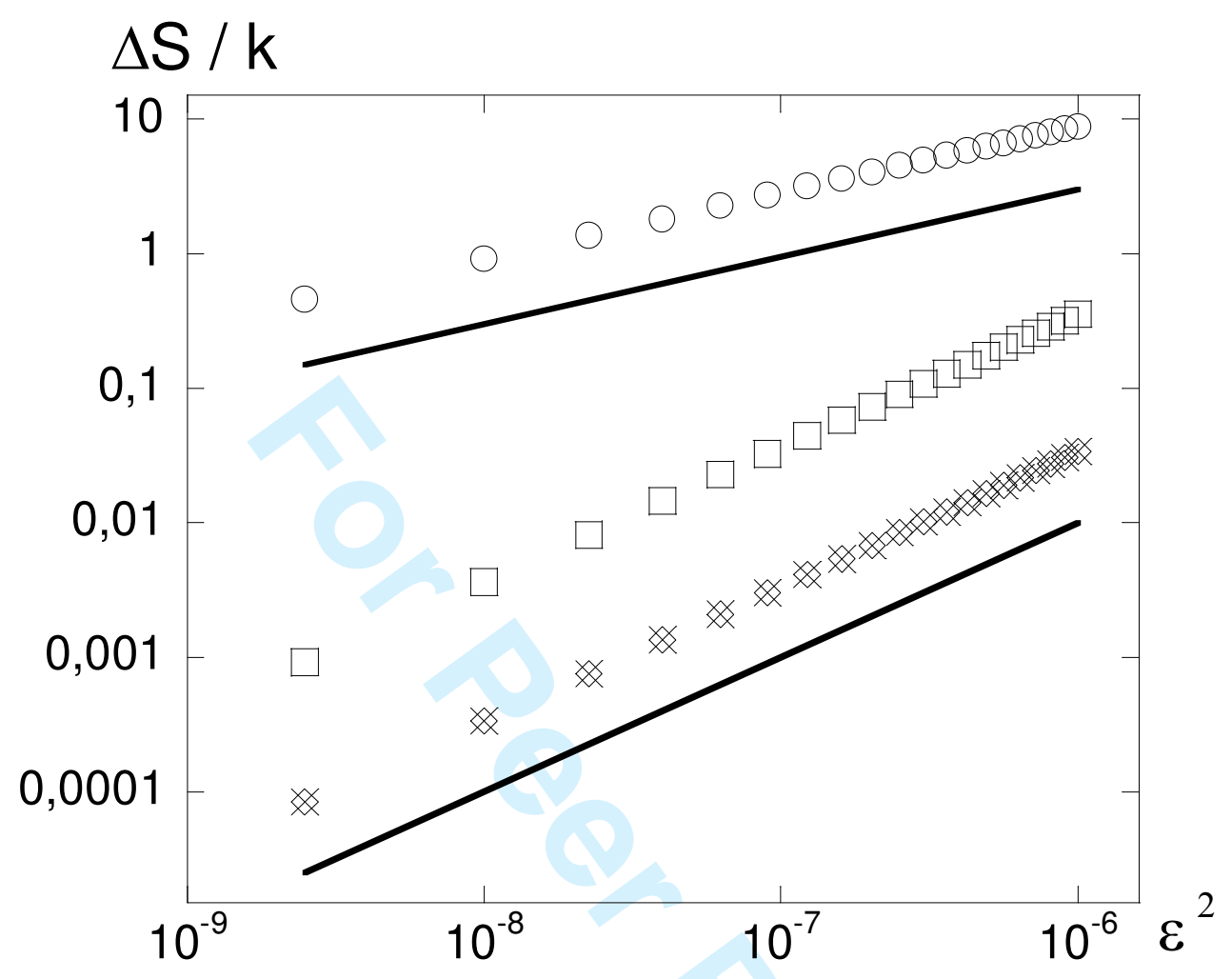

Figure 1 


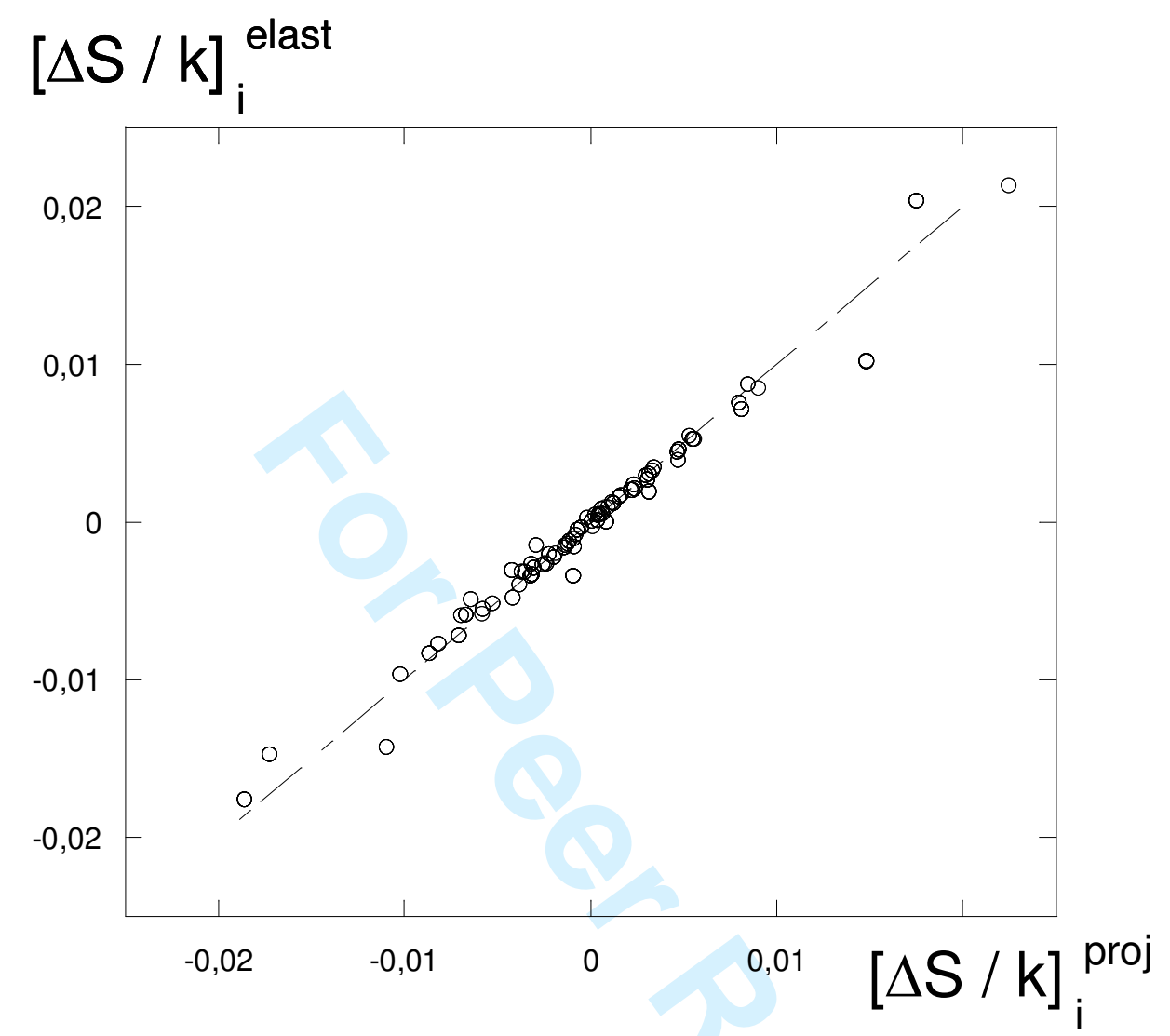

Figure 2 


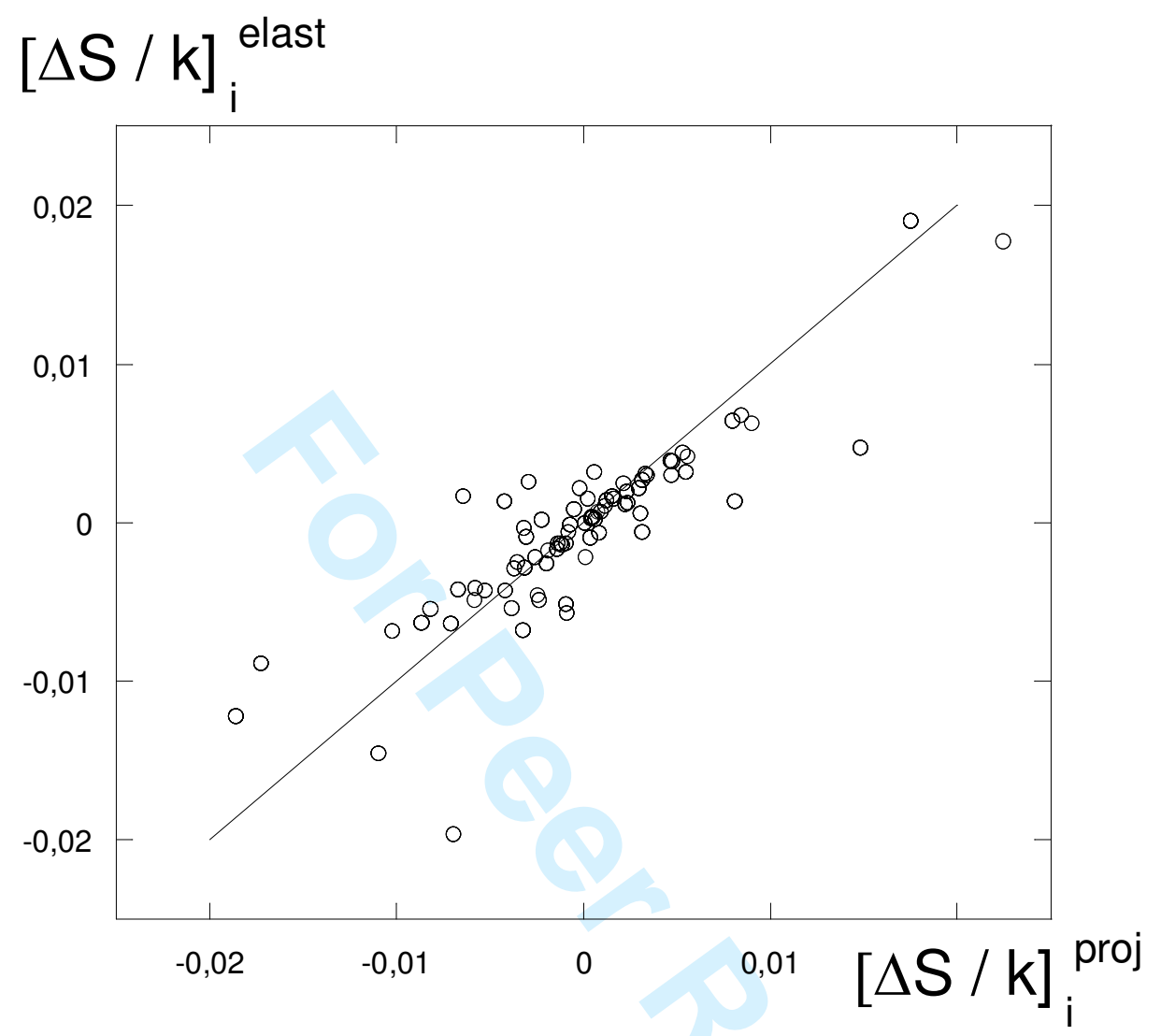

Figure 3 


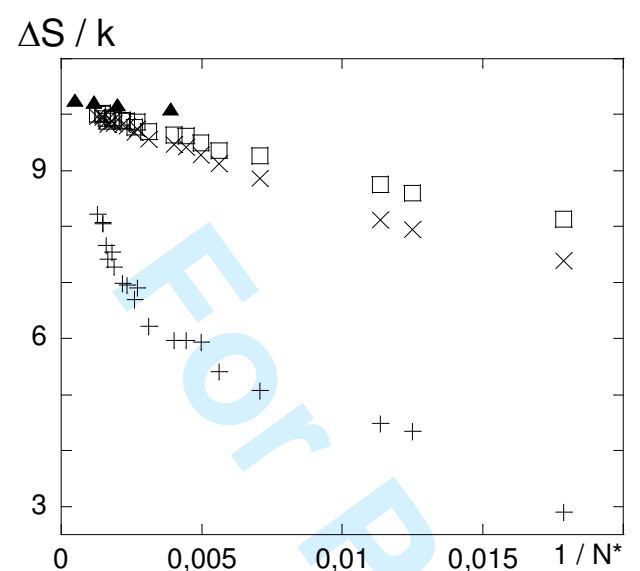

(a)

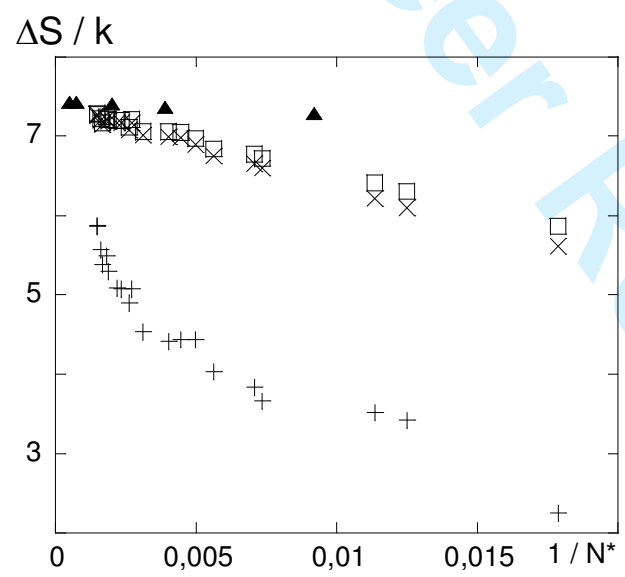

(c)

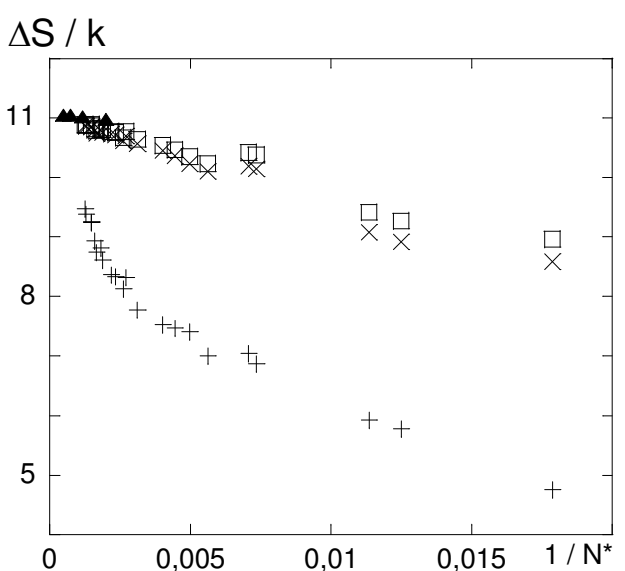

(b)

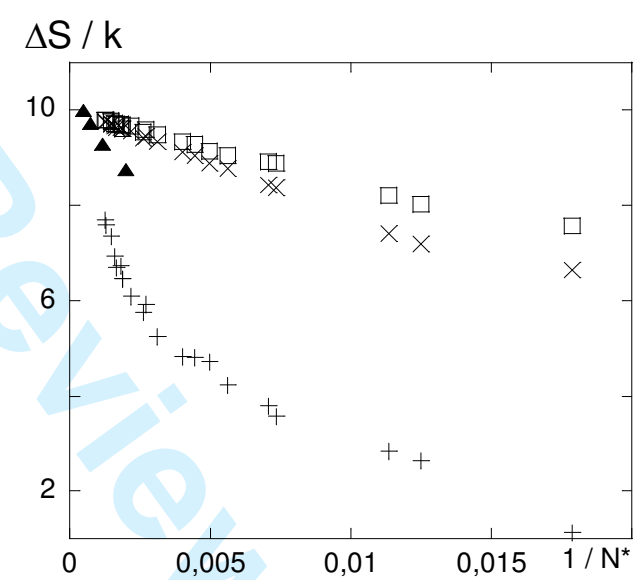

(d)

Figure 4 


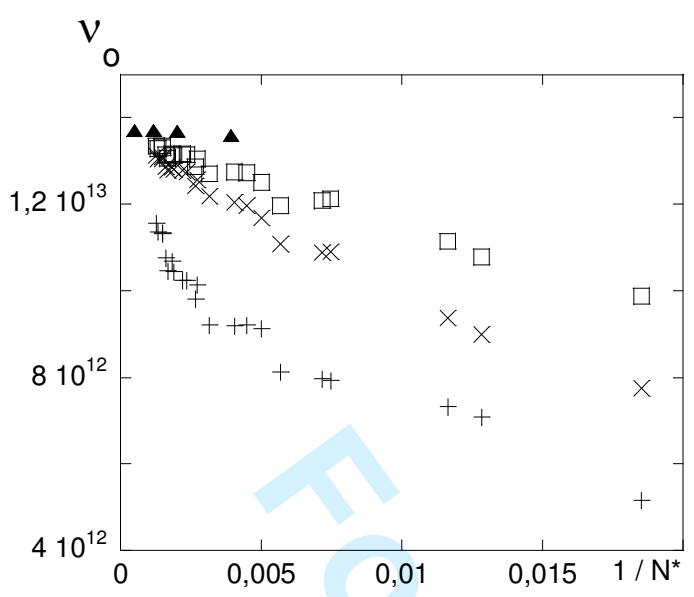

(a)

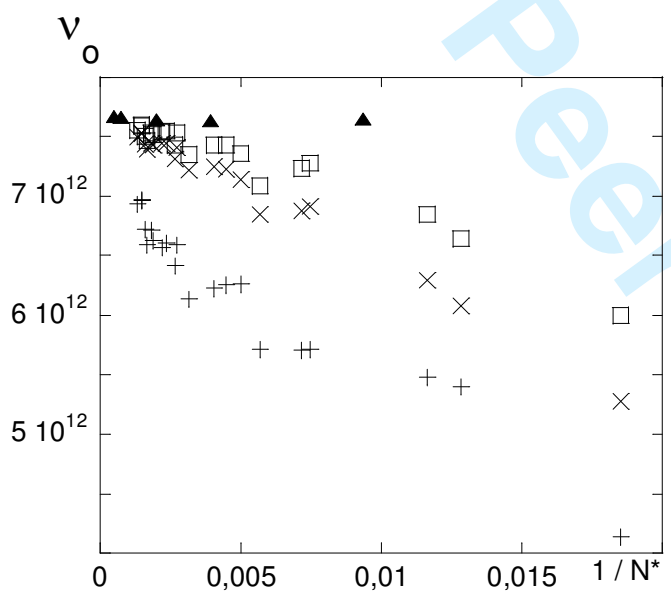

(c)

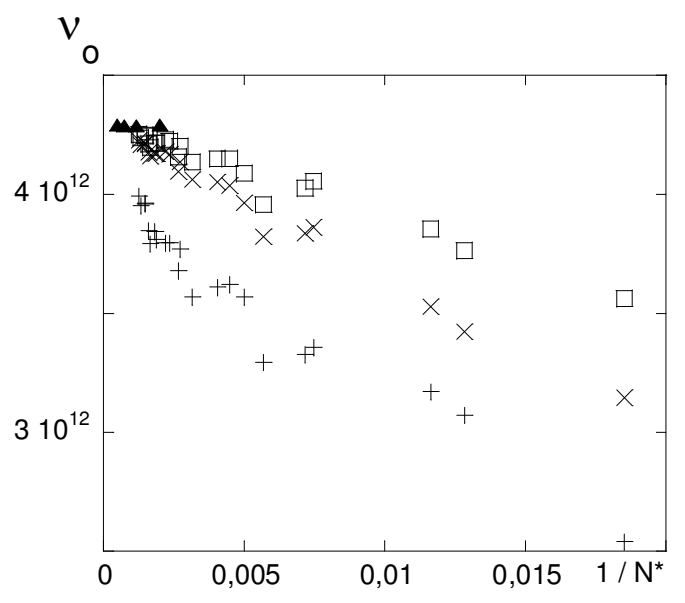

(b)

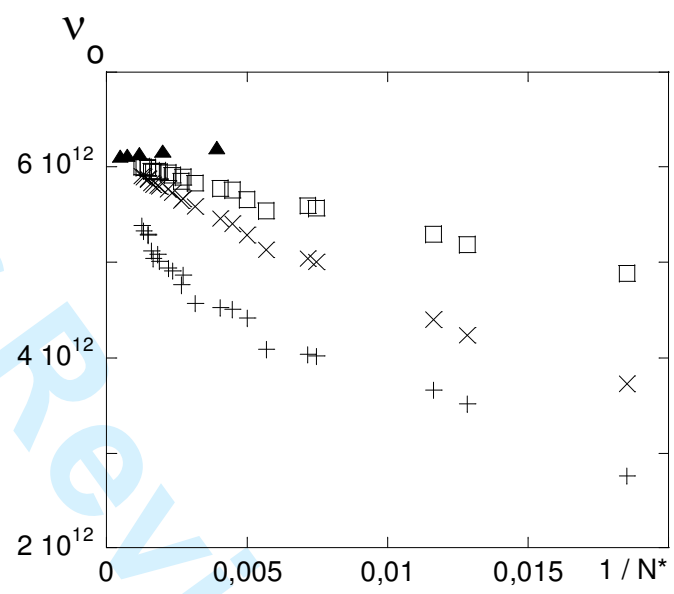

(d)

Figure 5 


1
2
3
4
5
6
7
8
9
10
11
12
13
14
15
16
17
18
19
20
21
22
23
24
25
26
27
28
29
30
31
32
33
34
35
36
37
38
39
40
41
42
43
40
45
49
50
51
52
53
55
50

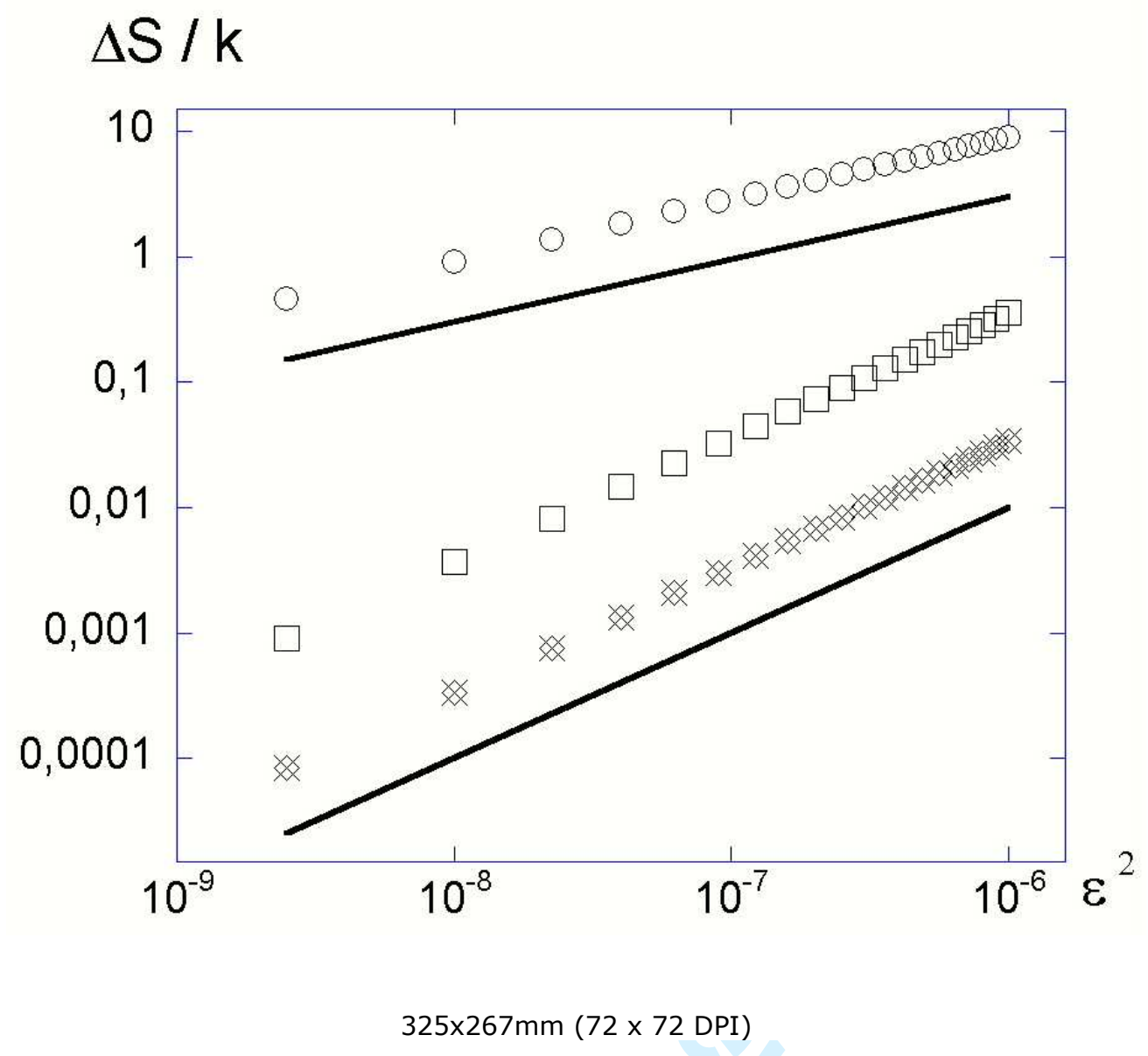

http://mc.manuscriptcentral.com/pm-pml 


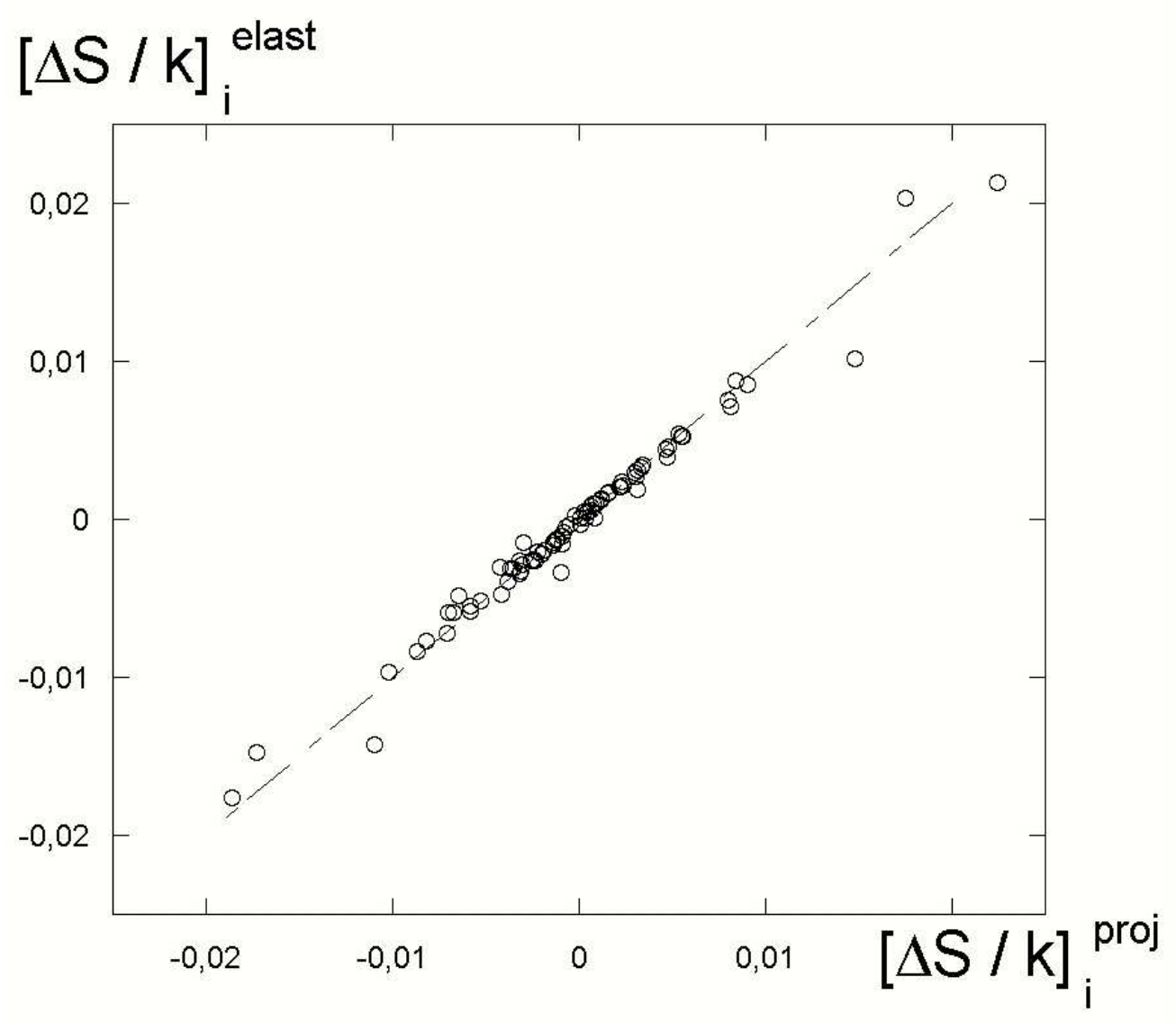

$319 \times 285 \mathrm{~mm}(72 \times 72 \mathrm{DPI})$

http://mc.manuscriptcentral.com/pm-pml 


1
2
3
4
5
6
7
8
9
10
11
12
13
14
15
16
17
18
19
20
21
22
23
24
25
26
27
28
29
30
31
32
33
34
35
36
37
38
39
40
41
42
43
40
45
49
50
51
52
53
55
50

$[\Delta \mathrm{S} / \mathrm{k}]_{\mathrm{i}}^{\text {elast }}$

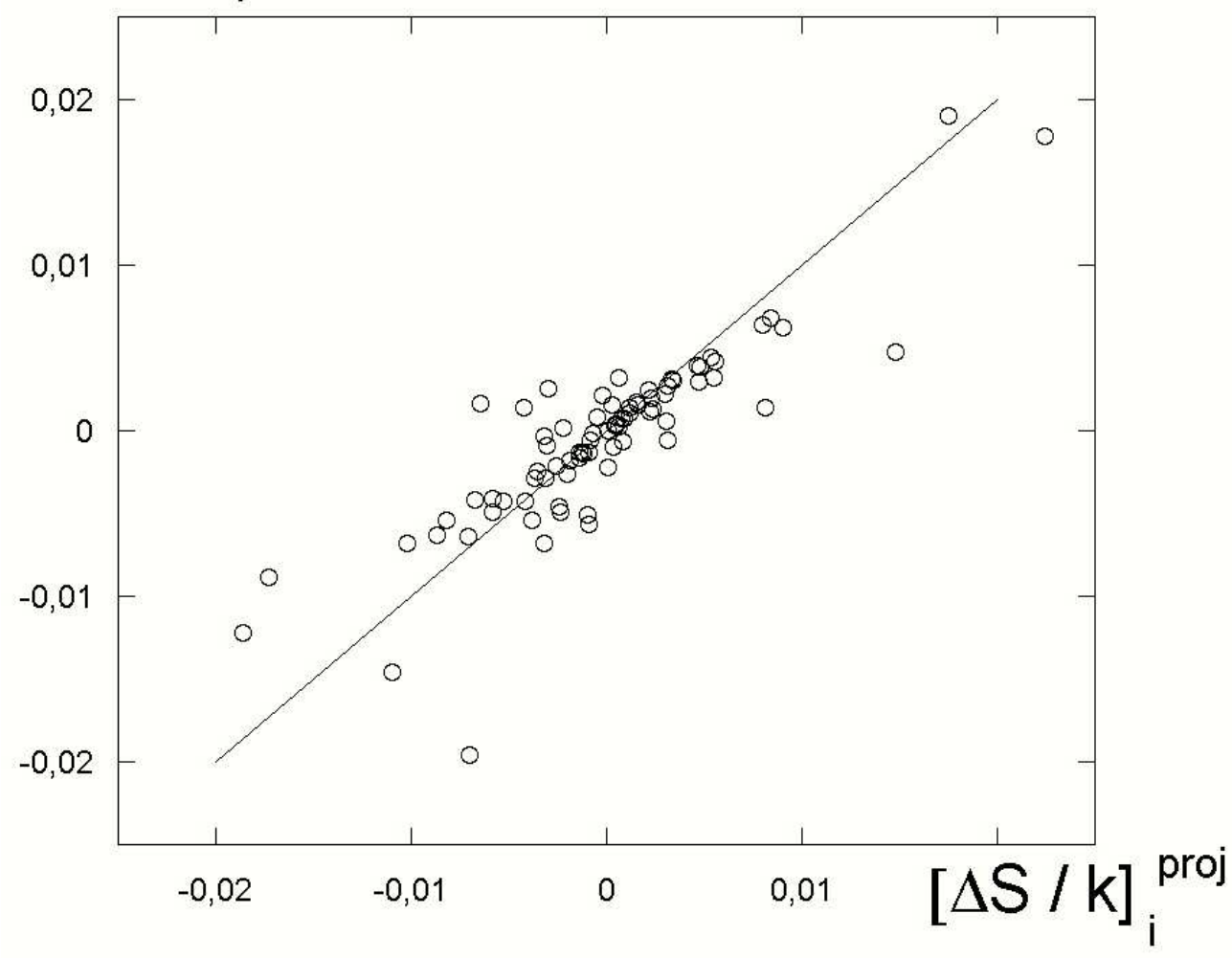

$323 \times 287 \mathrm{~mm}(72 \times 72 \mathrm{DPI})$ 


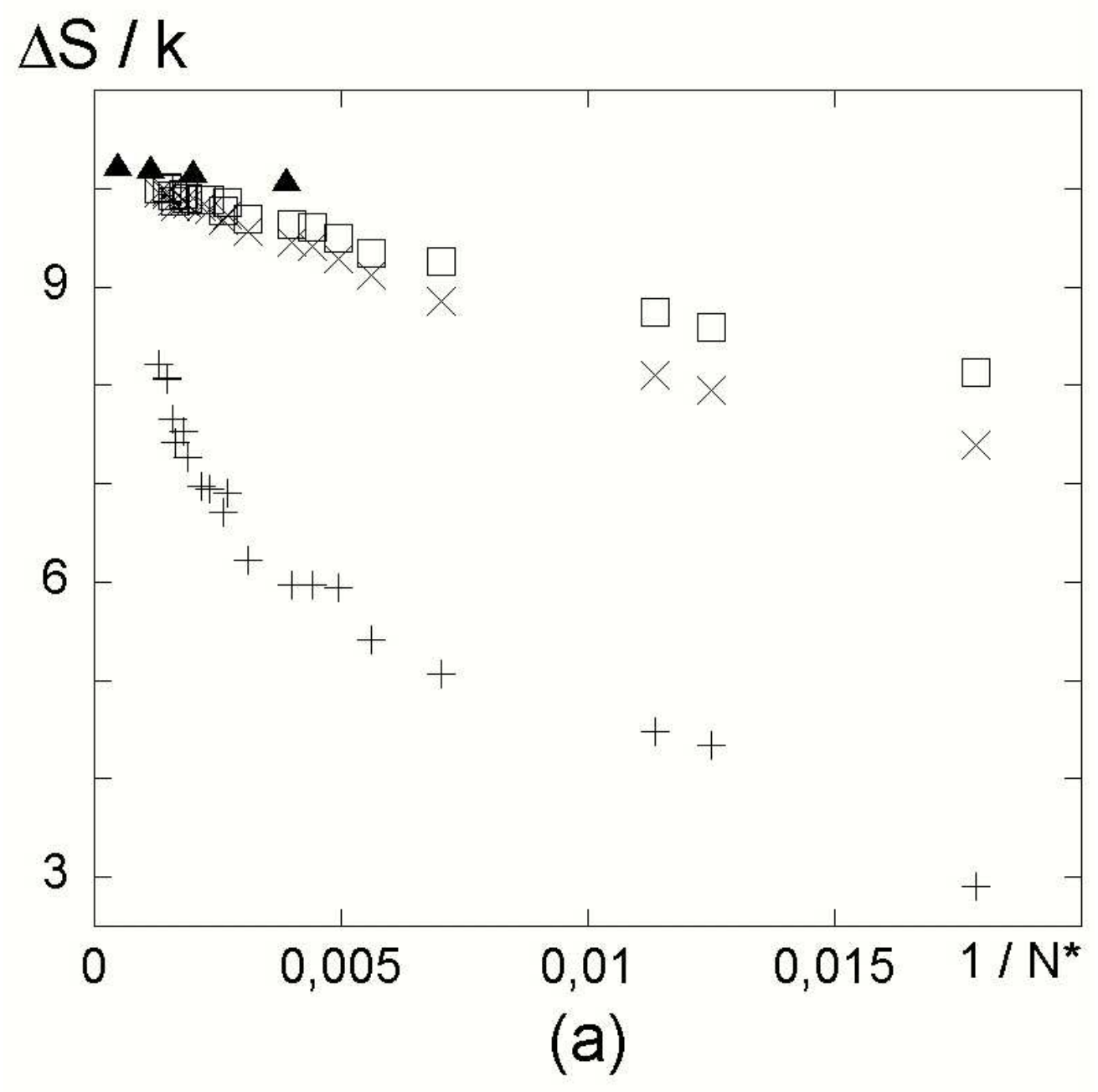

$280 \times 281 \mathrm{~mm}(72 \times 72$ DPI $)$ 


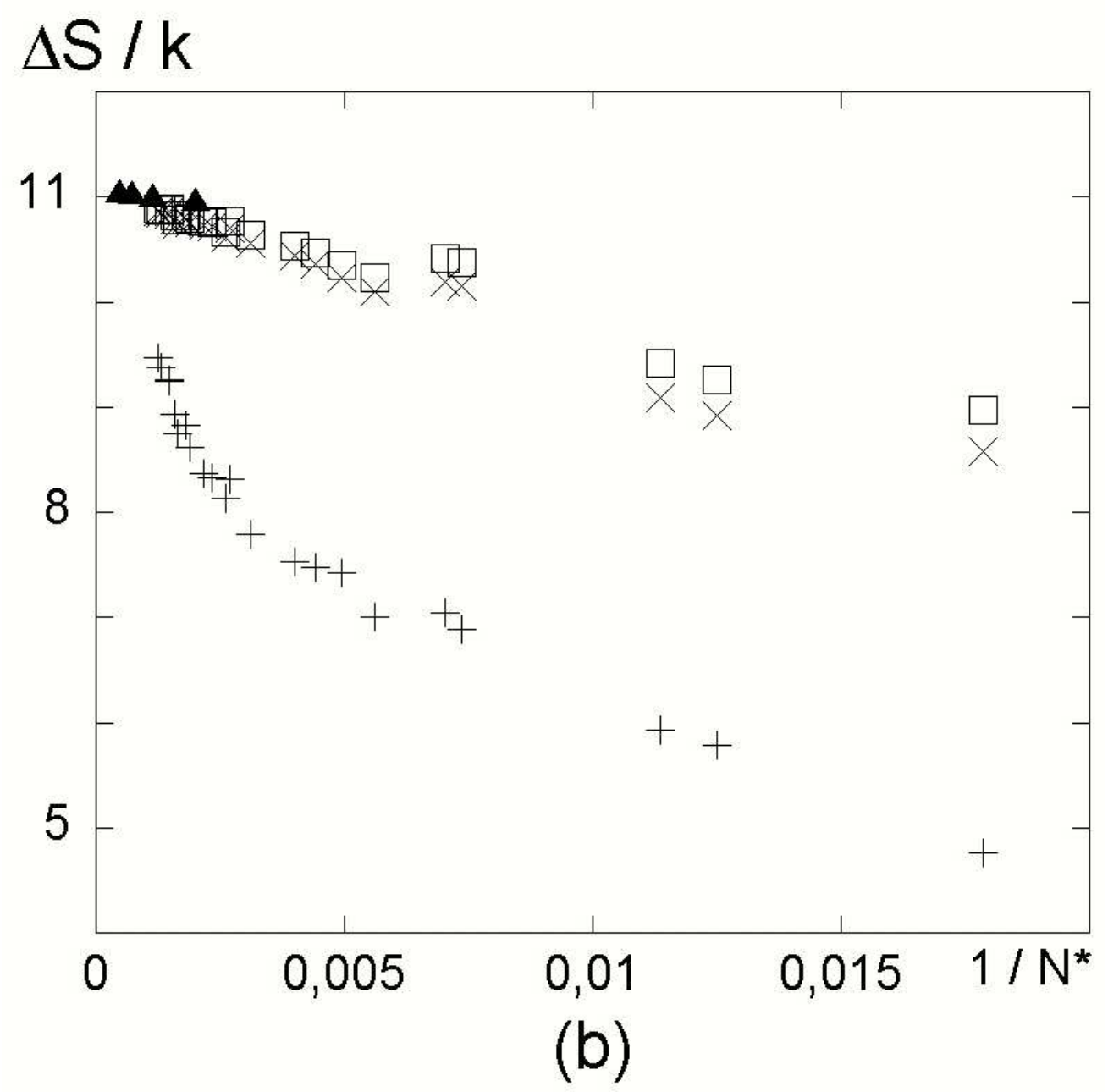

$282 \times 281 \mathrm{~mm}(72 \times 72 \mathrm{DPI})$ 


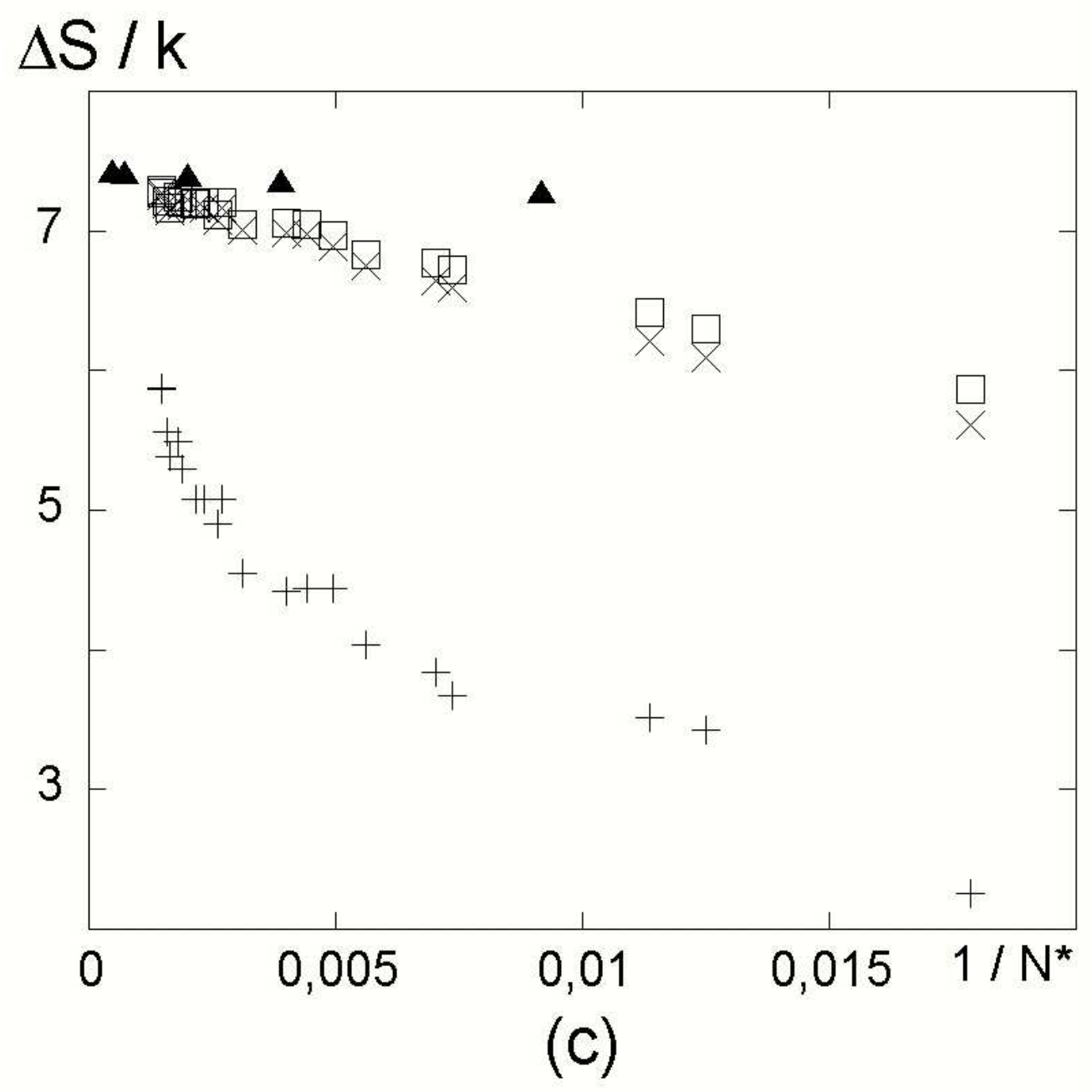

$280 \times 281 \mathrm{~mm}(72 \times 72$ DPI $)$

http://mc.manuscriptcentral.com/pm-pml 


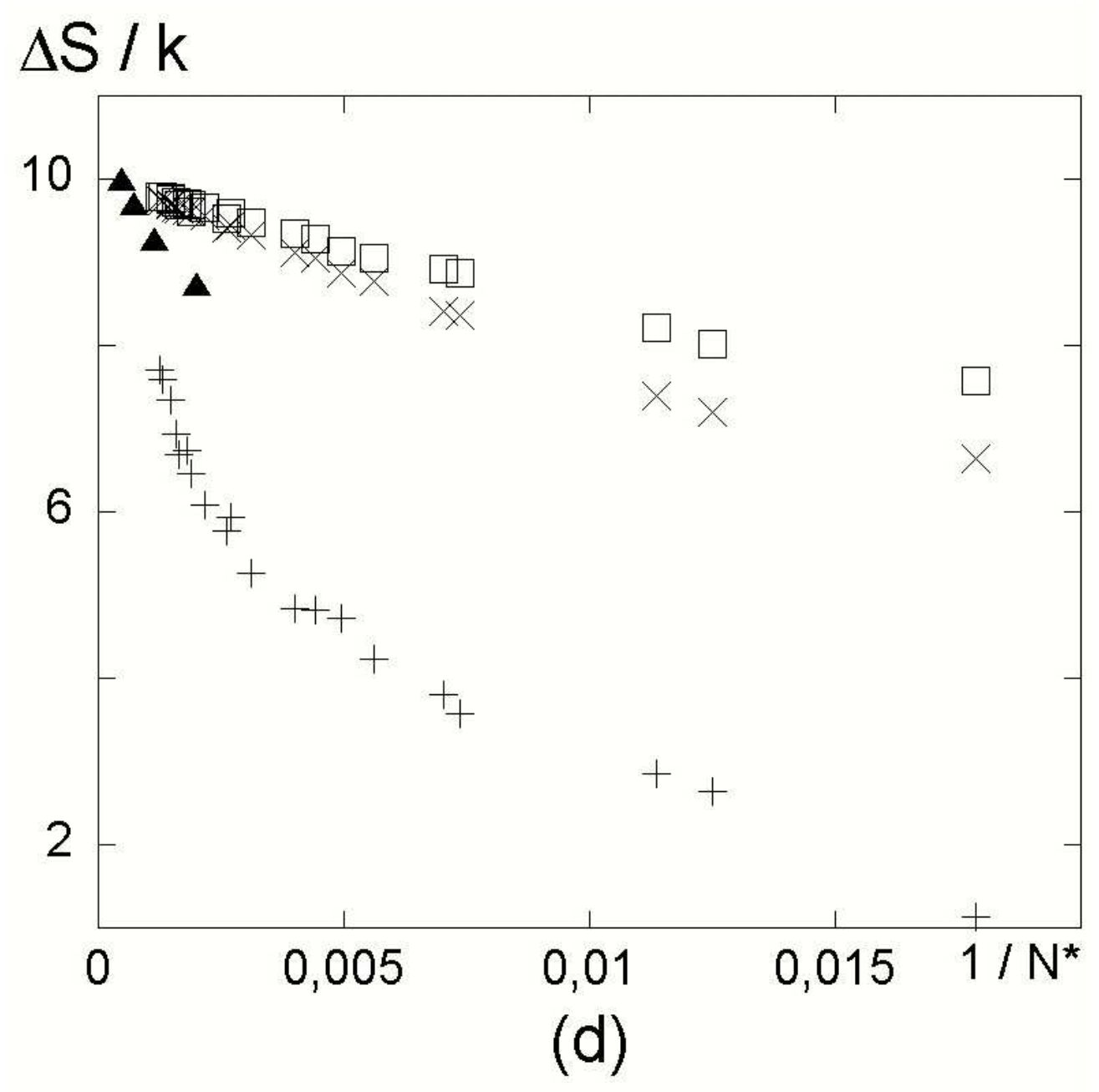

$281 \times 282 \mathrm{~mm}(72 \times 72 \mathrm{DPI})$ 


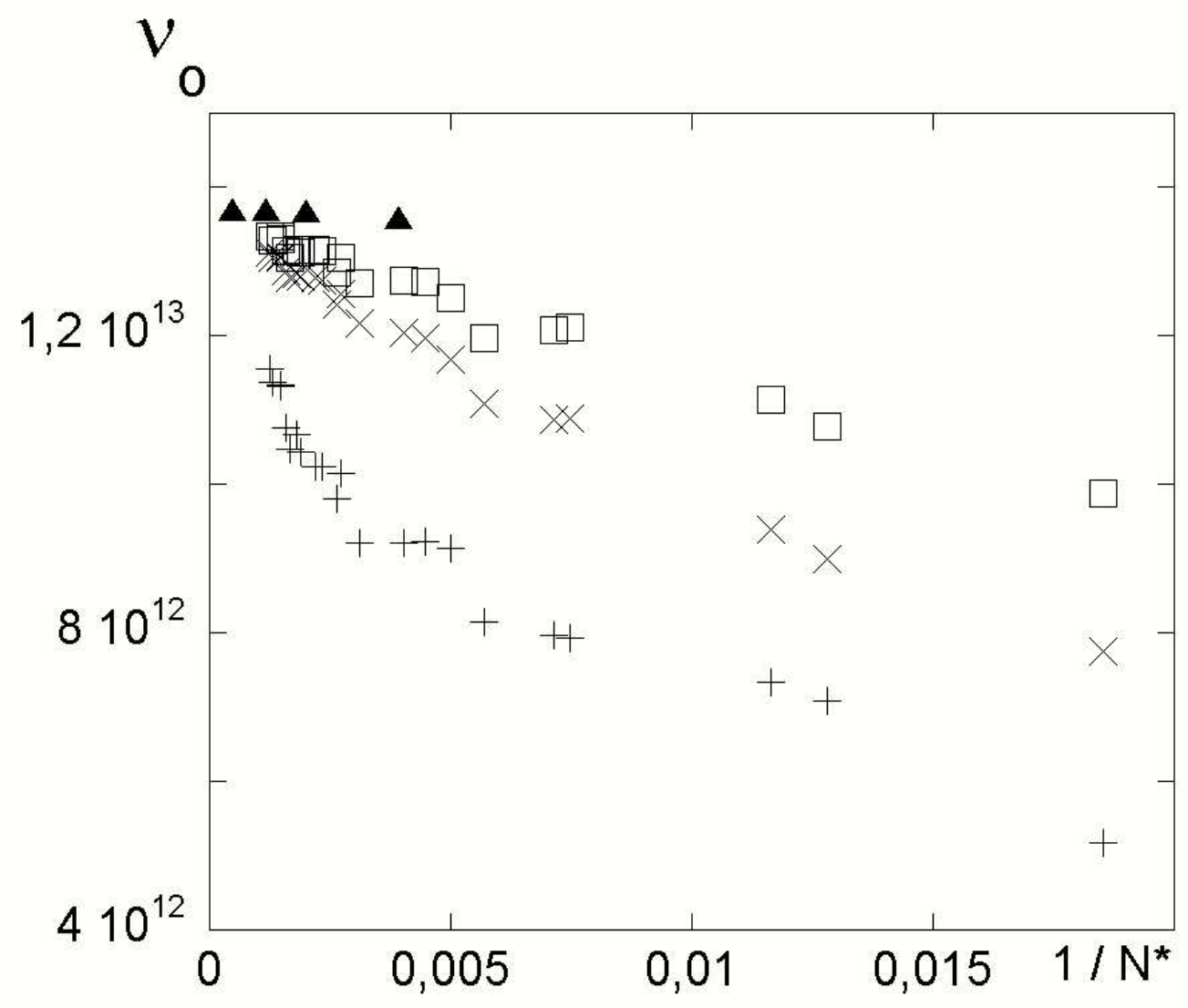

(a)

$312 \times 295 \mathrm{~mm}(72 \times 72 \mathrm{DPI})$ 


1
2
3
4
5
6
7
8
9
10
11
12
13
14
15
16
17
18
19
20
21
22
23
24
25
26
27
28
29
30
31
32
33
34
35
36
37
38
39
40
41
42
43
40
45
49
50
51
52
53
55
50

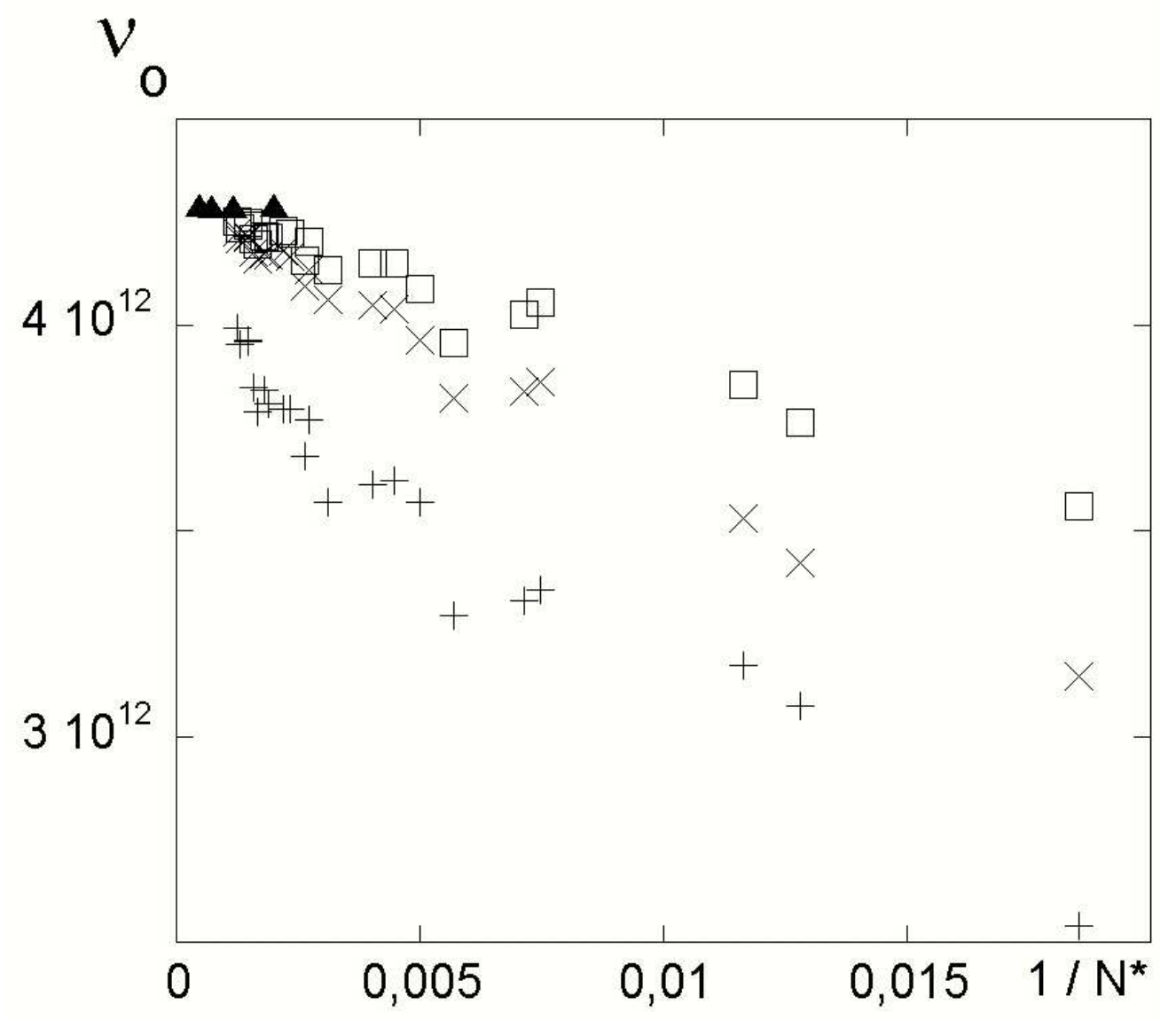

(b)

$301 \times 296 \mathrm{~mm}(72 \times 72 \mathrm{DPI})$ 


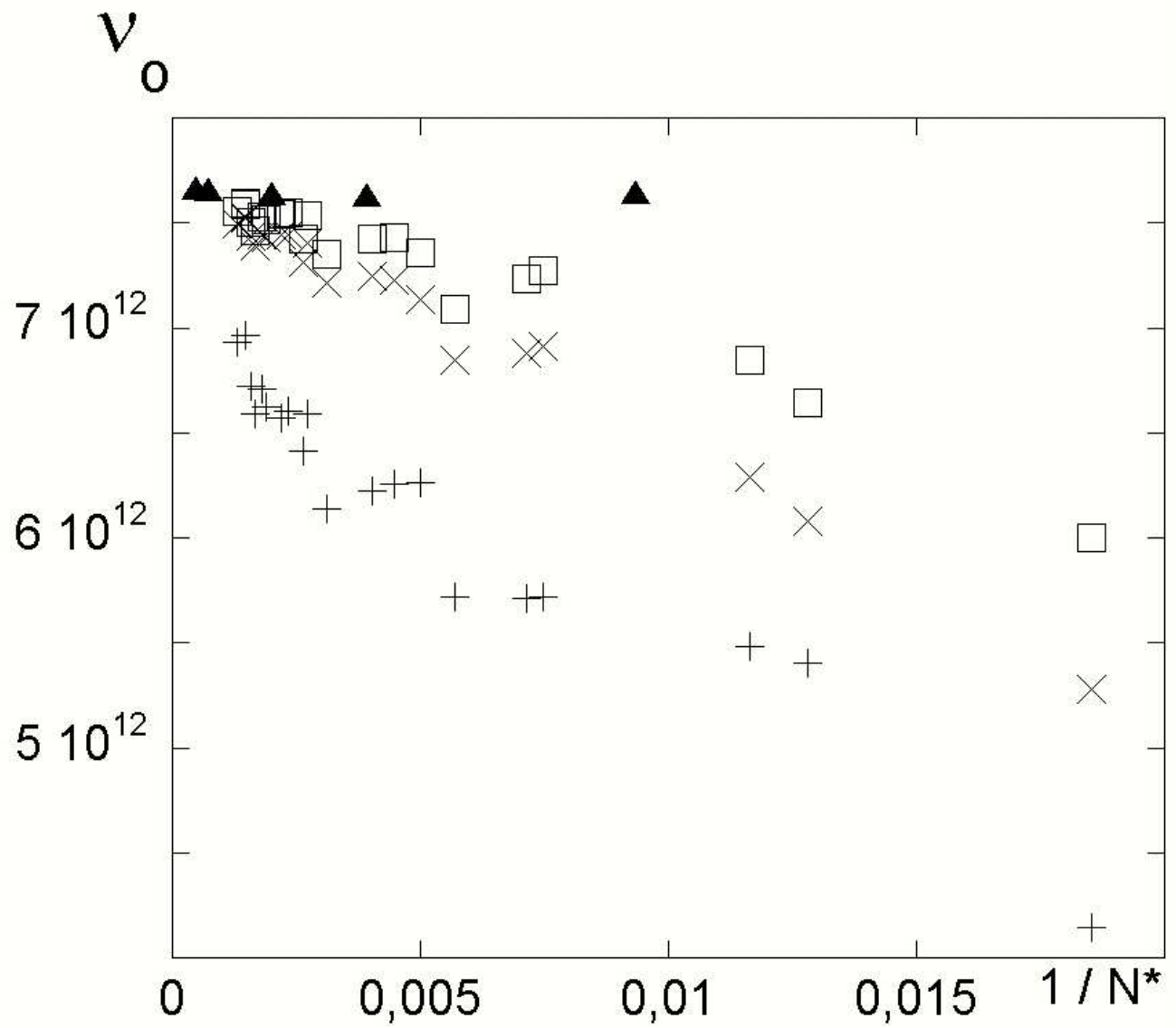

(c)

$301 \times 292 \mathrm{~mm}(72 \times 72$ DPI $)$ 


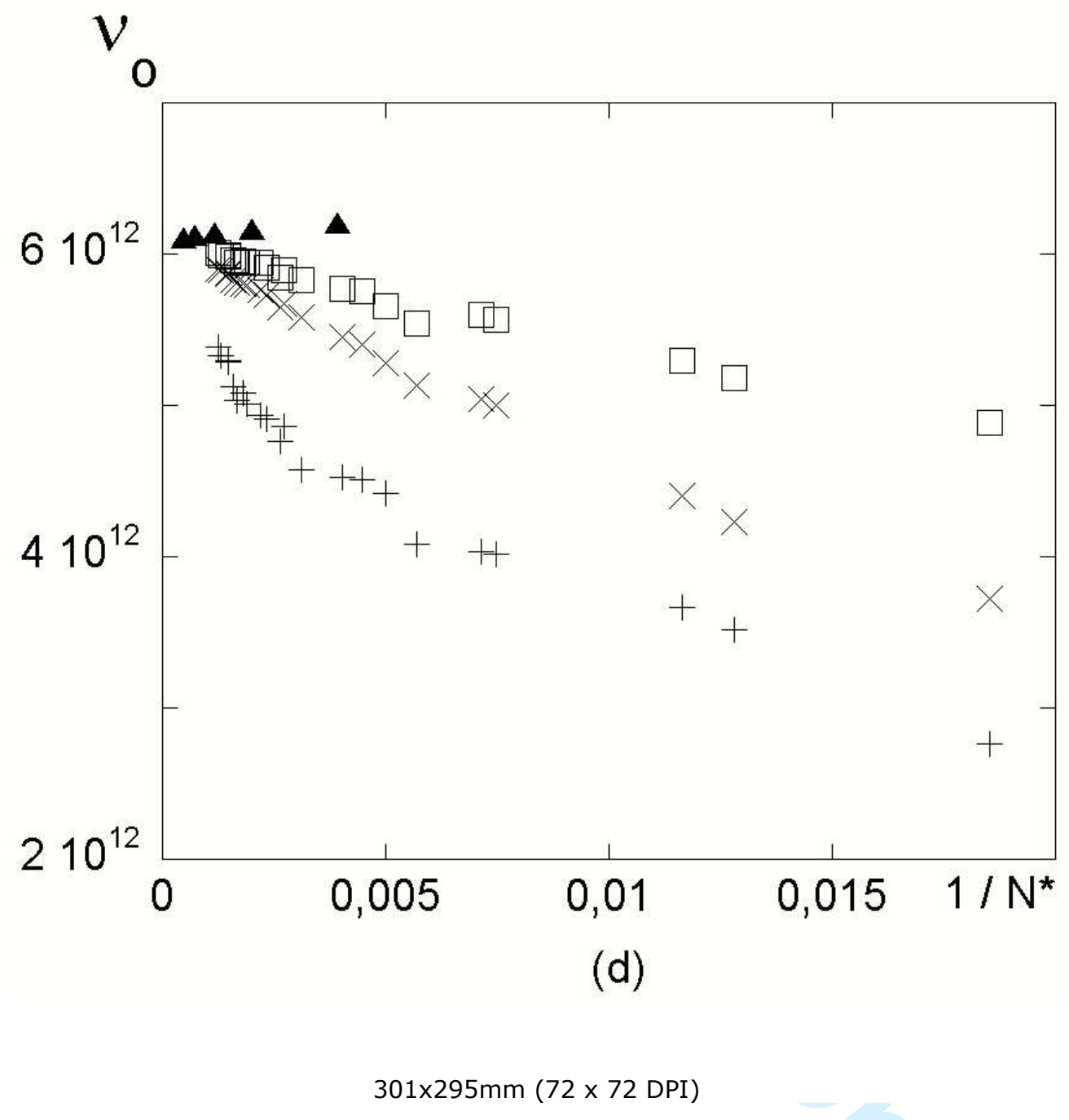

1

2

3

4

5

6
7

8

9

10

11

12

13

14

15

16

17

18

19

20

21

22

23

24

25

26

27

28

29

30

31

32

33

34

35

36

37

38

39

40

41

42

43

44

45

46

47

48

49

50

51

52

53

54

55

56

57

58

59

60

http://mc.manuscriptcentral.com/pm-pml 